\title{
Discontinuous Galerkin finite element method for shallow two-phase flows
}

\author{
S. Rhebergen*, O. Bokhove and J.J.W. van der Vegt \\ Department of Applied Mathematics, University of Twente, P.O. Box 217, 7500 \\ AE, Enschede, The Netherlands
}

\begin{abstract}
We present a discontinuous Galerkin finite element method for a depth-averaged two-phase flow model. This model contains nonconservative products for which we developed a discontinuous Galerkin finite element formulation in Rhebergen et al. (2008) J. Comput. Phys. 227, 1887-1922. The goal is to qualitatively validate the model against a laboratory experiment and to show the abilities of the model to capture physical phenomena. To be able to perform these test cases, a WENO slope limiter is investigated in conjunction with a discontinuity detector to detect regions where spurious oscillations appear.
\end{abstract}

Key words: discontinuous Galerkin finite element methods, multiphase flows, nonconservative products, slope limiter, discontinuity detector PACS: 02.60.Cb, 02.70.Dh, 47.55.-t

1991 MSC: 35L65, 35Q35, 65M60, 76M10, 76T99

\section{Introduction}

Debris flows are flows of water-saturated slurry mixtures (Iverson and Denlinger [14], Major and Iverson [22], Pitman and Le [25]). Examples are mud slides initiated by heavy rainfall on eroded mountain sides consisting of mixtures of rock, sand and mud; and volcanic debris flows in which the flow may be a mixture of volcanic debris and water (see Fig. 1 a). These flows often cause major destruction to buildings and infrastructure, with accompanying loss of human lives.

\footnotetext{
* Corresponding author. Tel.: +31 (0)53 4893415; fax: +31 (0)53 4894833

Email addresses: rhebergens@math.utwente.nl (S. Rhebergen),

o.bokhove@math. utwente.nl (O. Bokhove),

j.j.w.vandervegt@math.utwente.nl (J.J.W. van der Vegt).
} 


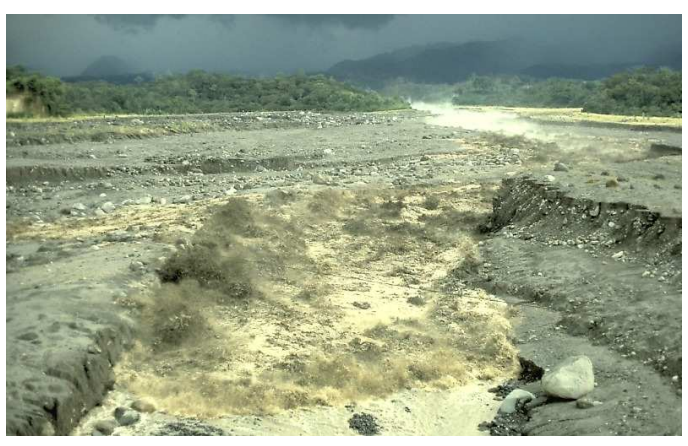

(a) The lahar developed on the slopes of Santiaguito volcano [37]. Photograph courtesy of U.S. Geological Survey.

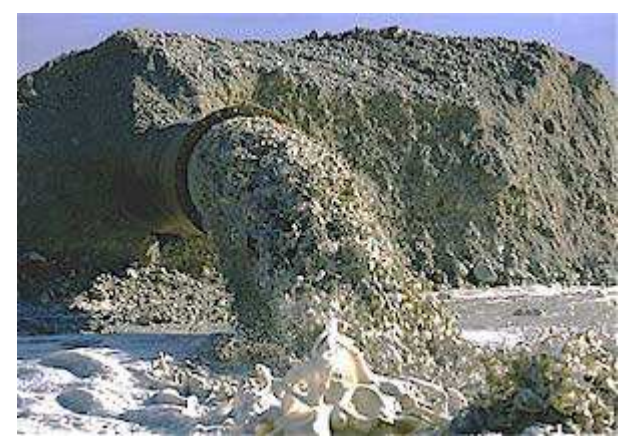

(b) Slurry and sediment transport in pipelines. Photograph courtesy of LICengineering [36].

Fig. 1. Examples of two-phase flows.

In industrial applications, dense liquid-solid flows, such as slurry flows, are used in pipeline transportation (see Fig. $1 \mathrm{~b}$ ). This form of transportation has relatively low operation and maintenance costs, and is friendly to the environment (Ling et al. [20]). Other applications occur in liquid fluidized beds (Jackson [15]).

In many flows the height $H$ of the flow is much smaller than the length $L$ of the flow, $H / L \ll 1$. For these flows, depth-averaging techniques are commonly used to simplify the three dimensional equations. Examples include the shallow water equations derived from the incompressible Navier-Stokes equations or the Savage-Hutter equations for dry granular flow (Savage and Hutter [28]). Recently, Pitman and Le [25] and Le [18] derived a depth-averaged model for two-phase flows based on a three dimensional continuum model for two-phase flows as derived by Jackson [15] (see Appendix A for the three dimensional continuum model). We remark, however, that with the assumptions made in the depth-averaging proces by Le [18], the same depth-averaged model can be derived from the three dimensional model of Drew and Lahey [10]. We have slightly extended the depth-averaged model by also including extra friction terms to simulate turbulent friction.

For the depth-averaged two-phase flow model, we present a discontinuous Galerkin finite element method (DGFEM). Among other advantages, DGFEM is a very local scheme, i.e., the solution on an element depends only on the data of its immediate neighboring elements, it is therefore very suitable to use for complicated geometries and mesh adaptation. Furthermore, the DGFEM easily deals with shocks and other discontinuities in the solution. The difficulty in the depth-averaged two-phase flow model is the presence of nonconservative products so that this model cannot be written in flux conservative form. This causes problems once the solution becomes discontinuous, because the weak solution in the classical sense of distributions then does not exist. Consequently, 
no classical Rankine-Hugoniot shock conditions can be defined. We overcame these problems in Rhebergen et al. [27], where we introduced a discontinuous Galerkin finite element method and a new numerical flux, the NCP flux, for hyperbolic partial differential equations containing nonconservative products which is based on the theory by Dal Maso, LeFloch and Murat (DLM) [8], which we also apply here.

The DGFEM does not guarantee monotone solutions around discontinuities and sharp gradients and thus spurious numerical oscillations develop. To prevent these numerical oscillations we investigate and clarify the WENO slope limiter given in [21] in combination with Krivodonova's discontinuity detector [17].

Much of the research conducted with depth averaged models for liquid-solid flows focuses on correctly predicting the final depositions of debris avalanches and their behavior over natural terrains (Denlinger and Iverson [9], Patra et al. [23,24], Pouliquen and Forterre [26], Tai et al. [29], Wang et al. [34]). In Chiou et al. [6] and Gray et al. [11] the influence of obstacles on granular flows is investigated. We are, however, interested in the behavior of debris flows through contractions and in this article we will perturb a steady-state two-phase flow with a low particle volume fraction by introducing an upstream avalanche of particles for a short period, thus temporarily increasing the particle volume fraction. This experiment was done by Akers and Bokhove [1] (see Fig. 2) and we use this experiment to qualitatively validate the depth-averaged two-phase flow model. Experimental data are also available for dry granular flow through a contraction (Vreman et al. [33]). We are planning to conduct new experiments to obtain data for liquid-solid flows through a contraction with which the numerical data may be compared in the future.

The novelties in this article are the following:

(1) Application of the discontinuous Galerkin finite element method using the theory of nonconservative products developed in [27] to the twodimensional depth-averaged two-phase flow model of Le [18] with extra friction terms.

(2) Investigation of the WENO slope limiter [21] with Krivodonova's discontinuity detector [17] in a discontinuous Galerkin finite element discretization for two-phase flows.

(3) Qualitative validation of the depth-averaged two-phase flow model with laboratory data.

The outline of this article is as follows. In Section 2 we present the depthaveraged model as derived by Pitman and Le [25] and Le [18]. We continue in Section 3 to introduce the discontinuous Galerkin finite element method for the model; numerical verification and validation is provided in Sections 4 

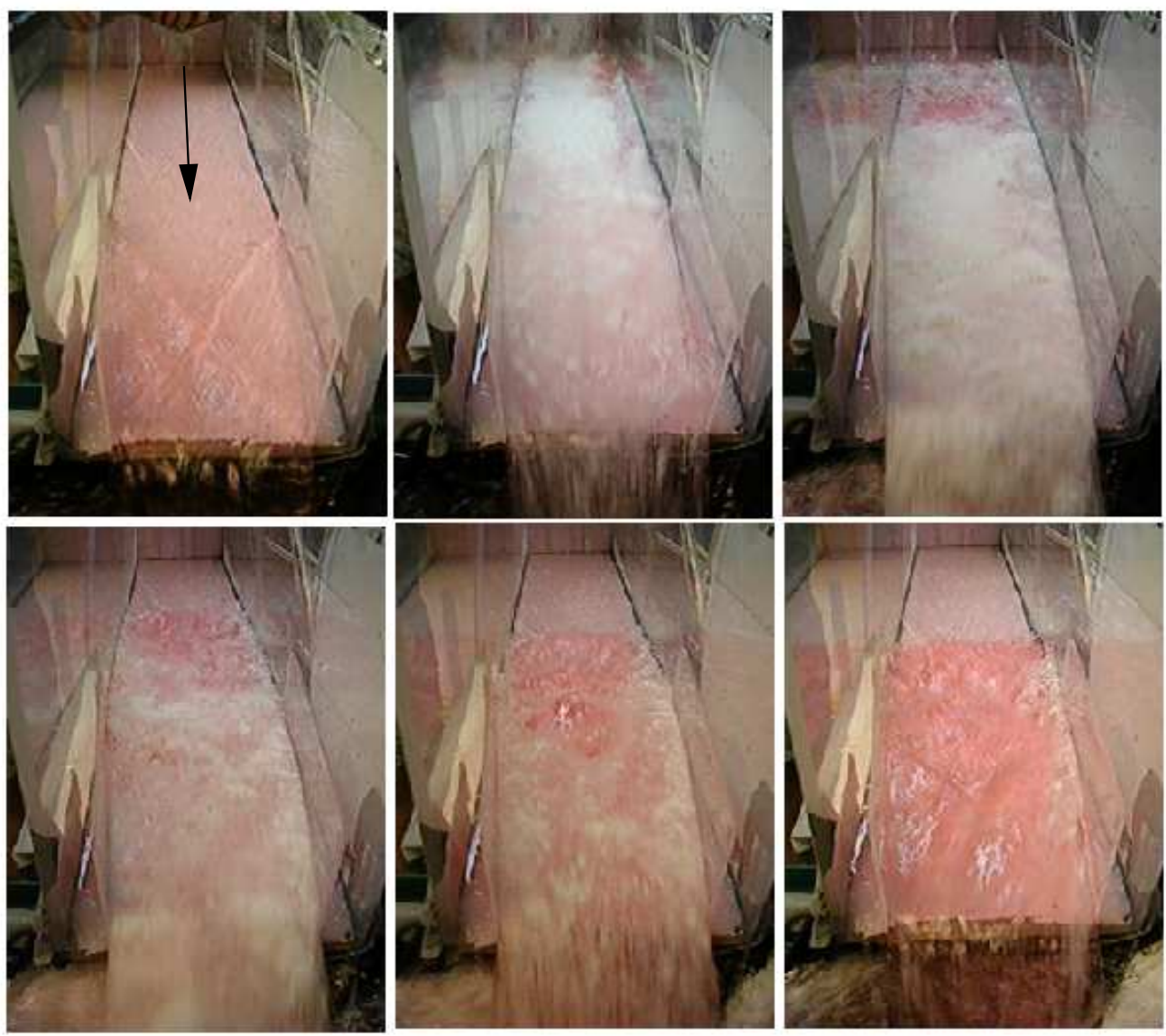

Fig. 2. When water flow enters a contraction at a certain speed, a steady state in the contraction is reached with oblique hydraulic jumps (top left). This steady-state is perturbed by an upstream avalanche of polystyrene beads (just inserted in the top middle frame). There is a transition period in the top right, bottom left and bottom middle frames in which one second elapses between each frame. A second steady state, an upstream steady shock, is reached (bottom right) [1].

and 5. Conclusions are drawn in Section 6.

\section{Depth-averaged two-phase flows}

In shallow flows, the characteristic height $H$ of the flow is typically much smaller than its characteristic length $L, H / L=\varepsilon \ll 1$. Variations in the vertical are small and we can simplify the governing equations by averaging the flow over the depth. In doing so, depth-averaged quantities are assumed to be independent of the vertical coordinate, at leading order in $\varepsilon$. In this section we introduce the depth-averaged two-phase flow equations derived by Le [18]. Note that the depth-averaged two-phase flow equations derived by Le [18] are slightly different from the depth-averaged two-phase flow equations derived 
by Pitman and Le [25]. The difference is that the momentum of the mixture of the Le model can be written in flux conservative form, while this is not the case for the momentum of the mixture of the Pitman and Le model.

Le [18] derived a depth-averaged flow model by depth-averaging the three dimensional continuum model for two-phase flows as derived by Jackson [15] (see Appendix A for the three dimensional model). Using the summation convention on repeated indices and the comma notation to denote partial differentiation, the scaled non-dimensional depth-averaged flow model is:

$$
U_{i, t}+F_{i k, k}+G_{i k r} V_{r, k}=S_{i}, \quad i, r=1, \ldots, 6, k=1,2 .
$$

Note that $G_{i k r} V_{r, k}$ is a nonconservative product. In (1) $U=\left[h(1-\alpha), h \alpha, h \alpha v_{i}, h(1-\right.$ $\left.\alpha) u_{i}\right]^{T}, V=\left[h, \alpha, v_{i}, u_{i}\right]^{T}$ and

$$
\begin{gathered}
F_{k}=\left[\begin{array}{c}
h(1-\alpha) u_{k} \\
h \alpha v_{k} \\
h \alpha v_{i} v_{k}+\varepsilon(1-\rho) \varphi_{i k} \alpha \frac{1}{2} g_{3} h^{2} \\
h(1-\alpha) u_{i} u_{k}
\end{array}\right], \quad G_{k}=\left[\begin{array}{cccc}
0 & 0 & 0 & 0 \\
0 & 0 & 0 & 0 \\
\varepsilon \rho \alpha g_{3} h & 0 & 0 & 0 \\
\varepsilon(1-\alpha) g_{3} h & 0 & 0 & 0
\end{array}\right], \\
0 \\
S=\left[\begin{array}{c}
0 \\
(1-\rho)\left(-\varepsilon \varphi_{i k} \partial_{k} b+\varphi_{i 3}\right) \alpha g_{3} h+h F_{i}^{D}+g_{i} h \alpha-\rho \alpha C_{D}|u| u_{i} / \varepsilon-\varepsilon \rho h \alpha g_{3} \partial_{i} b \\
-\varepsilon(1-\alpha) g_{3} h \partial_{i} b-h F_{i}^{D} / \rho+h(1-\alpha) g_{i}-(1-\alpha) C_{D}|u| u_{i} / \varepsilon
\end{array}\right] .
\end{gathered}
$$

Note that compared with the model by Le [18], we have added extra friction terms with the drag coefficient $C_{D}$ as a leading order turbulence parameterization.

The orientation of the Cartisian coordinate system is shown in Figure 3 in which $\theta$ is the angle of the $x_{1}-x_{2}$ plane with the horizontal. The depth-averaged quantities in the above model are constant in the $x_{3}$ direction and are the particle volume fraction $\alpha$, the fluid velocity vector $u$ and the solids velocity vector $v$. The flow depth is given by $h$ and the bottom topography by $b$. The constants $\varepsilon=H / L$ and $\rho=\rho^{f} / \rho^{s}$ represent the height to length ratio of the flow and the ratio between the fluid density $\rho^{f}$ and the solids density $\rho^{s}$, respectively. The gravity vector is given by $\vec{g}=\left[g_{1}, g_{2},-g_{3}\right]^{T}$ in which $g_{3}$ is the vertical component of the gravity (see Figure 3 ) and $C_{D}$ is a drag coefficient. The above quantities are all scaled and dimensionless. To obtain the variables in dimensional form, denoted by $(\cdot)^{*}$, we have used the following scalings: $\left[x^{*}, y^{*}\right]=L[x, y], t^{*}=\sqrt{L / g^{*}} t,\left[u_{1}^{*}, u_{2}^{*}\right]=\sqrt{g^{*} L}\left[u_{1}, u_{2}\right],\left[v_{1}^{*}, v_{2}^{*}\right]=\sqrt{g^{*} L}\left[v_{1}, v_{2}\right]$, $v_{T}^{*}=\sqrt{g^{*} L} v_{T},\left[g_{1}^{*}, g_{2}^{*}, g_{3}^{*}\right]^{T}=g^{*} g[\sin (\theta), 0, \cos (\theta)]^{T}$ with $g$ the gravity constant. 


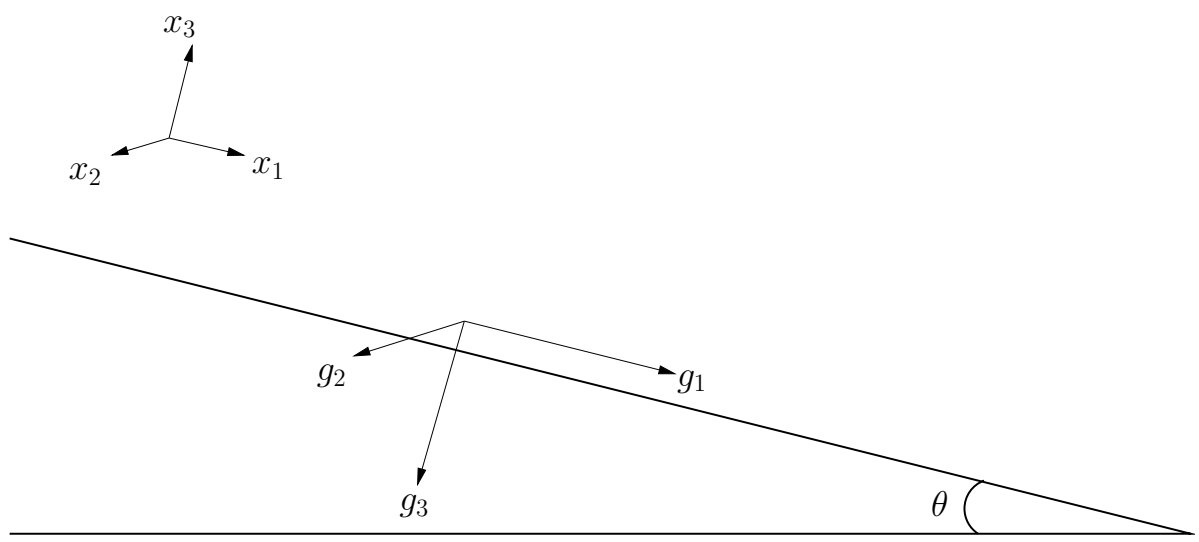

Fig. 3. Orientation of the coordinate system and the gravity vector.

A closure needs to be given for the drag function $F^{D}$ and we follow Pitman and Le [25] by taking $F_{i}^{D}=\beta\left(u_{i}-v_{i}\right)$ in which $\beta$ is given by:

$$
\beta=\frac{(1-\rho) \alpha}{v_{T}(1-\alpha)^{n}}, \quad n= \begin{cases}3.65 & \text { for } R e_{t}<0.2 \\ 4.35 R e_{t}^{-0.03}-1 & \text { for } 0.2<R e_{t}<1 \\ 4.45 R e_{t}^{-0.1}-1 & \text { for } 1<R e_{t}<500 \\ 1.39 & \text { for } 500<R e_{t}\end{cases}
$$

where $R e_{t}=d \rho_{f} v_{T} / \mu_{f}$, in which $d$ is the particle diameter, $\rho^{f}$ the fluid density, $\mu_{f}$ the fluid viscosity and $v_{T}$ the terminal velocity of an isolated particle falling in the fluid. We remark that as $1-\rho$ increases, the drag function $F^{D}$ makes the system (1) increasingly stiffer. We are, however, interested in the case where $\rho$ is approximately 0.9 . In this situation the model does not have stiff source terms and no special algorithms are needed to deal with stiffness.

The functions $\varphi$ were introduced by Pitman and Le [25] to relate basal and diagonal shear stresses to the normal stress in the solids phase stress tensor in the 3-dimensional two-phase model before depth-averaging. The functions $\varphi$ are given by:

$$
\begin{aligned}
\varphi_{i 3} & =-\frac{v_{i}}{\|v\|} \tan \left(\phi_{\text {bed }}\right), \quad i=1,2, \quad \varphi_{i i}=k^{\mp}, \quad i=1,2 \\
\varphi_{12} & =-\operatorname{sign}\left(\partial_{2} v_{1}\right) \sin \left(\phi_{\text {int }}\right) k^{\mp}, \quad \varphi_{21}=-\operatorname{sign}\left(\partial_{1} v_{2}\right) \sin \left(\phi_{\text {int }}\right) k^{\mp}, \\
k^{\mp} & =2 \frac{1 \mp \sqrt{1-\cos ^{2}\left(\phi_{\text {int }}\right)\left(1+\tan ^{2}\left(\phi_{\text {bed }}\right)\right)}}{\cos ^{2}\left(\phi_{\text {int }}\right)}-1,
\end{aligned}
$$

in which the "-" in the "F" applies when $\partial_{k} v_{k}>0$ and the "+" applies when $\partial_{k} v_{k}<0$. Furthermore, $\|\cdot\|$ is the Euclidean norm, $\phi_{\text {int }}$ is the internal angle of friction, which measures how layers of solid particles slide over one another and $\phi_{\text {bed }}$ is the basal angle of friction, indicating how easily solid particles slide 

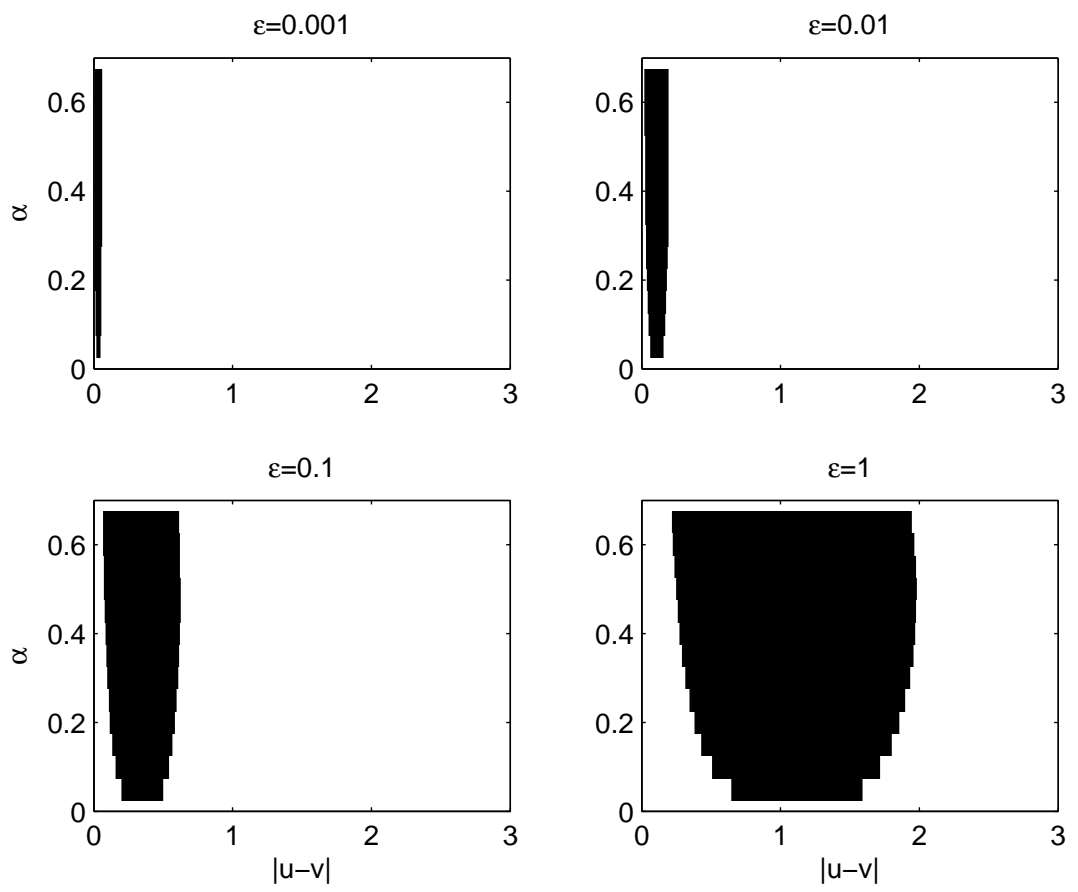

Fig. 4. Regimes of hyperbolicity for the depth-averaged model. For the values of $\alpha$ and $|u-v|$ in the shaded area the model is elliptic.

over the bottom [13].

To determine whether the depth averaged model is hyperbolic, we need to determine their eigenvalues. If all eigenvalues are real and distinct, the model is hyperbolic. Deriving the eigenvalues for the depth-averaged model is not trivial, so eigenvalues are computed numerically for a number of given parameters. Consider the case in which the topography is flat, $b=0$. We take $h=1$, $\rho=0.9, g_{3}=1$ and we assume $k^{\mp}=k^{-}$. Furthermore, we take $\phi_{\text {bed }}=14.75^{\circ}$ and $\phi_{\text {int }}=24.5^{\circ}$ which hold for fine glass particles [4]. For different height to length ratios, ranging from $\varepsilon=0.001$ to $\varepsilon=1$, we determine the eigenvalues as a function of the particle volume fraction $\alpha$ and the absolute difference between the phase velocities $|u-v|$. In Figure 4 we show for which values of $\alpha$ and $|u-v|$ the depth-averaged model is not hyperbolic (in the shaded areas some of the eigenvalues are not real). We see that the region for which the model is not hyperbolic decreases as $\varepsilon$ decreases. In this article we are only interested in cases where the model is hyperbolic. When the model is not hyperbolic, a different numerical approach needs to be introduced which is not treated in this article. 


\section{The DGFEM discretization}

In this section we present a space-time DGFEM formulation for the depthaveraged two-phase flow model. We remark however that the space DGFEM formulation is very similar and for some of the numerical test cases we will apply space DGFEM. For more on space DGFEM we refer to Rhebergen et al. [27] and Cockburn and Shu [7].

We start by introducing space-time elements, function spaces, trace operators and basis functions after which we present the space-time DGFEM formulation for the depth-averaged two-phase flow model.

\subsection{Space-time elements}

In the space-time DGFEM method, the space and time variables are treated together. A point at time $t=x_{0}$ with position vector $\bar{x}=\left(x_{1}, x_{2}\right)$ has Cartesian coordinates $\left(x_{0}, \bar{x}\right)$ in the open domain $\mathcal{E} \subset \mathbb{R}^{3}$. At time $t$, the flow domain $\Omega(t)$ is defined as:

$$
\Omega(t):=\left\{\bar{x} \in \mathbb{R}^{2}:(t, \bar{x}) \in \mathcal{E}\right\} .
$$

By taking $t_{0}$ and $T$ as the initial and final time of the evolution of the spacetime flow domain, the space-time domain boundary $\partial \mathcal{E}$ consists of the hypersurfaces:

$$
\begin{aligned}
\Omega\left(t_{0}\right) & :=\left\{x \in \partial \mathcal{E}: x_{0}=t_{0}\right\} \\
\Omega(T) & :=\left\{x \in \partial \mathcal{E}: x_{0}=T\right\} \\
\mathcal{Q} & :=\left\{x \in \partial \mathcal{E}: t_{0}<x_{0}<T\right\} .
\end{aligned}
$$

The time interval $\left[t_{0}, T\right]$ is partitioned using the time levels $t_{0}<t_{1}<\ldots<T$, where the $n$-th time interval is defined as $I_{n}=\left(t_{n}, t_{n+1}\right)$ with length $\Delta t_{n}=$ $t_{n+1}-t_{n}$. The space-time domain $\mathcal{E}$ is then divided into $N_{t}$ space-time slabs $\mathcal{E}^{n}=\mathcal{E} \cap I_{n}$. Each space-time slab $\mathcal{E}^{n}$ is bounded by $\Omega\left(t_{n}\right), \Omega\left(t_{n+1}\right)$ and $\mathcal{Q}^{n}=\partial \mathcal{E}^{n} /\left(\Omega\left(t_{n}\right) \cup \Omega\left(t_{n+1}\right)\right)$.

The flow domain $\Omega\left(t_{n}\right)$ is approximated by $\Omega_{h}\left(t_{n}\right)$, where $\Omega_{h}(t) \rightarrow \Omega(t)$ as $h \rightarrow 0$, with $h$ the radius of the smallest sphere completely containing the largest space-time element. The domain $\Omega_{h}\left(t_{n}\right)$ is divided into $N_{n}$ nonoverlapping spatial elements $K_{j}\left(t_{n}\right)$. Similarly, $\Omega\left(t_{n+1}\right)$ is approximated by $\Omega_{h}\left(t_{n+1}\right)$. We can relate each element $K_{j}^{n}=K_{j}\left(t_{n}\right)$ to a master element $\hat{K} \subset \mathbb{R}^{2}$ through the mapping $F_{K}^{n}$ :

$$
F_{K}^{n}: \hat{K} \rightarrow K_{j}^{n}: \bar{\xi} \mapsto \bar{x}=\sum_{i} x_{i}\left(K_{j}^{n}\right) \chi_{i}(\bar{\xi})
$$


with $x_{i}$ the spatial coordinates of the vertices of the spatial element $K_{j}^{n}$ and $\chi_{i}$ the standard Lagrangian shape functions defined on element $\hat{K}$. The spacetime elements $\mathcal{K}_{j}^{n}$ are constructed by connecting $K_{j}^{n}$ with $K_{j}^{n+1}$ using linear interpolation in time, resulting in the mapping $G_{\mathcal{K}}^{n}$ from the master element $\hat{\mathcal{K}} \subset \mathbb{R}^{3}$ to the space-time element $\mathcal{K}^{n}$ :

$$
\begin{aligned}
G_{\mathcal{K}}^{n}: \hat{\mathcal{K}} \rightarrow \mathcal{K}^{n}: \xi=\left(\xi_{0}, \bar{\xi}\right) \mapsto(t, \bar{x})= & \left(\frac{1}{2}\left(t_{n+1}+t_{n}\right)+\frac{1}{2}\left(t_{n+1}-t_{n}\right) \xi_{0},\right. \\
& \left.\frac{1}{2}\left(1-\xi_{0}\right) F_{K}^{n}(\bar{\xi})+\frac{1}{2}\left(1+\xi_{0}\right) F_{K}^{n+1}(\bar{\xi})\right),
\end{aligned}
$$

with $\xi_{0} \in[-1,1]$ and $\bar{\xi} \in \hat{K}$. The tessellation $\mathcal{T}_{h}^{n}$ of the space-time slab $\mathcal{E}_{h}^{n}$ consists of all space-time elements $\mathcal{K}_{j}^{n}$; thus the tessellation $\mathcal{T}_{h}$ of the discrete flow domain $\mathcal{E}_{h}:=\cup_{n=0}^{N_{t}-1} \mathcal{E}_{h}^{n}$ then is defined as $\mathcal{T}_{h}:=\cup_{n=0}^{N_{t}-1} \mathcal{T}_{h}^{n}$.

The element boundary $\partial \mathcal{K}_{j}^{n}$, the union of open faces of $\mathcal{K}_{j}^{n}$, consists of three parts: $K_{j}\left(t_{n}^{+}\right)=\lim _{\epsilon \downarrow 0} K_{j}\left(t_{n}+\epsilon\right), K_{j}\left(t_{n+1}^{-}\right)=\lim _{\epsilon \downarrow 0} K_{j}\left(t_{n+1}-\epsilon\right)$ and $\mathcal{Q}_{j}^{n}=$ $\partial \mathcal{K}_{j}^{n} /\left(K_{j}\left(t_{n}^{+}\right) \cup K_{j}\left(t_{n+1}^{-}\right)\right)$. The outward space-time normal vector on $\partial \mathcal{K}_{j}^{n}$ is given by:

$$
n= \begin{cases}(1, \overline{0}) & \text { at } K_{j}\left(t_{n+1}^{-}\right) \\ (-1, \overline{0}) & \text { at } K_{j}\left(t_{n}^{+}\right), \\ (0, \bar{n}) & \text { at } \mathcal{Q}_{j}^{n}\end{cases}
$$

where $\overline{0} \in \mathbb{R}^{2}$. It is convenient to split the element boundaries into separate faces. In addition to faces $K_{j}\left(t_{n}^{+}\right)$and $K_{j}\left(t_{n+1}^{-}\right)$, we also define therefore interior and boundary faces. An interior face is shared by two neighboring elements $\mathcal{K}_{i}^{n}$ and $\mathcal{K}_{j}^{n}$, such that $\mathcal{S}_{i j}^{n}=\mathcal{Q}_{i}^{n} \cap \mathcal{Q}_{j}^{n}$; a boundary face is defined as $\mathcal{S}_{B j}^{n}=\partial \mathcal{E}^{n} \cap \mathcal{Q}_{j}^{n}$. The set of interior faces in a space-time slab $\mathcal{E}^{n}$ is denoted by $\mathcal{S}_{I}^{n}$ and the set of all boundary faces by $\mathcal{S}_{B}^{n}$; the total set is denoted by $\mathcal{S}_{I, B}^{n}=\mathcal{S}_{I}^{n} \cup \mathcal{S}_{B}^{n}$.

\subsection{Function spaces and trace operators}

We consider approximations of $U(x, t)$ and functions $W(x, t)$ in the finite element space $W_{h}$ defined as:

$$
W_{h}=\left\{W \in\left(L^{2}\left(\mathcal{E}_{h}\right)\right)^{m}:\left.W\right|_{\mathcal{K}} \circ G_{\mathcal{K}} \in\left(P^{p}(\hat{\mathcal{K}})\right)^{m}, \forall \mathcal{K} \in \mathcal{T}_{h}\right\},
$$

where $L^{2}\left(\mathcal{E}_{h}\right)$ is the space of square integrable functions on $\mathcal{E}_{h}$ and $P^{p}(\hat{\mathcal{K}})$ the space of polynomials of degree at most $p$ on reference element $\hat{\mathcal{K}}$. Here $m$ denotes the dimension of $U$. In our case, $m=6$ and we use linear approximations with $p=1$.

We now introduce some operators as defined in Klaij et al. [16]. The trace 
of a function $f \in V_{h}$ at the element boundary $\partial \mathcal{K}^{L}$ is defined as:

$$
f^{L}=\lim _{\epsilon \downarrow 0} f\left(x-\epsilon n^{L}\right),
$$

with $n^{L}$ the unit outward space-time normal at $\partial \mathcal{K}^{L}$. When only the space components of the outward normal vector are considered we will use the notation $\bar{n}^{L}$. A function $f \in W_{h}$ has a double valued trace at element boundaries $\partial \mathcal{K}$. The traces of a function $f$ at an internal face $\mathcal{S}=\overline{\mathcal{K}}^{L} \cap \overline{\mathcal{K}}^{R}$ are denoted by $f^{L}$ and $f^{R}$. The jump of $f$ at an internal face $\mathcal{S} \in \mathcal{S}_{I}^{n}$ in the direction $k$ of a Cartesian coordinate system is defined as:

$$
\llbracket f \rrbracket_{k}=f^{L} \bar{n}_{k}^{L}+f^{R} \bar{n}_{k}^{R},
$$

with $\bar{n}_{k}^{R}=-\bar{n}_{k}^{L}$. The average of $f$ at $\mathcal{S} \in \mathcal{S}_{I}^{n}$ is defined as:

$$
\left\{\{f\}=\frac{1}{2}\left(f^{L}+f^{R}\right) .\right.
$$

The jump operator satisfies the following product rule at $\mathcal{S} \in \mathcal{S}_{I}^{n}$ for $\forall l \in W_{h}$ and $\forall f_{k} \in W_{h}$, which can be proven by direct verification:

$$
\llbracket l_{i} f_{i k} \rrbracket_{k}=\left\{\left\{l_{i}\right\} \llbracket \llbracket f_{i k} \rrbracket_{k}+\llbracket l_{i} \rrbracket_{k}\left\{\left\{f_{i k}\right\} .\right.\right.
$$

Consequently, we can relate element boundary integrals to face integrals:

$$
\sum_{\mathcal{K} \in \mathcal{T}_{h}^{n}} \int_{\mathcal{Q}} l_{i}^{L} f_{i k}^{L} \bar{n}_{k}^{L} d \mathcal{Q}=\sum_{\mathcal{S} \in \mathcal{S}_{I}^{n}} \int_{\mathcal{S}} \llbracket l_{i} f_{i k} \rrbracket_{k} d \mathcal{S}+\sum_{\mathcal{S} \in \mathcal{S}_{B}^{n}} \int_{\mathcal{S}} l_{i}^{L} f_{i k}^{L} \bar{n}_{k}^{L} d \mathcal{S} .
$$

\subsection{Basis functions}

Polynomial approximations for the trial function $U$ and the test functions $W$ in each element $\mathcal{K} \in \mathcal{T}_{h}^{n}$ are introduced as:

$$
\left.U(t, \bar{x})\right|_{\mathcal{K}}=\hat{U}_{m} \psi_{m}(t, \bar{x}) \text { and }\left.W(t, \bar{x})\right|_{\mathcal{K}}=\hat{W}_{l} \psi_{l}(t, \bar{x}),
$$

with $\psi_{m}$ the basis functions, $\bar{x} \in \mathbb{R}^{2}$, and expansion coefficients $\hat{U}_{m}$ and $\hat{W}_{l}$, respectively, for $m, l=0,1,2,3$. The basis functions $\psi_{m}$ are given by $\psi_{0}=1$ and $\psi_{m}=\varphi_{m}(t, \bar{x})$ for $m=1,2,3$ where the functions $\varphi_{m}(x)$ in element $\mathcal{K}$ are related to the basis functions $\hat{\varphi}_{m}(\xi)$, with $\hat{\varphi}_{m}(\xi) \in P^{1}(\hat{\mathcal{K}})$ and $\xi$ the local coordinates in the master element $\hat{\mathcal{K}}$, through the mapping $G_{\mathcal{K}}: \varphi_{m}=\hat{\varphi}_{m} \circ G_{\mathcal{K}}^{-1}$.

\subsection{The weak formulation}

Due to the nonconservative products (1) cannot be transformed into divergence form. This causes problems once the solution becomes discontinuous, 
because the weak solution in the classical sense of distributions then does not exist. Consequently, standard space-time DGFEM discretizations cannot be applied. In Rhebergen et al. [27] we derived a discontinuous Galerkin finite element weak formulation for general hyperbolic equations with nonconservative products and we apply this weak formulation here as well.

We refer to Rhebergen et al. [27] for the derivation of the weak formulation for (1). The main criterium posed on the weak formulation is that the formulation must reduce to that for the conservative system if the system of nonconservative partial differential equations can be transformed into conservative form. The weak formulation for (1) is given by:

Find a $U \in W_{h}$ such that for all $W \in W_{h}$ :

$$
\begin{aligned}
0= & \sum_{\mathcal{K} \in \mathcal{T}_{h}^{n}} \int_{\mathcal{K}}\left(-W_{i, 0} U_{i}-W_{i, k} F_{i k}+W_{i} G_{i k r} V_{r, k}-W_{i} S_{i}\right) d \mathcal{K} \\
& +\sum_{\mathcal{K} \in \mathcal{T}_{h}^{n}}\left(\int_{K\left(t_{n+1}^{-}\right)} W_{i}^{L} U_{i}^{L} d K-\int_{K\left(t_{n}^{+}\right)} W_{i}^{L} U_{i}^{R} d K\right) \\
& +\sum_{\mathcal{S} \in \mathcal{S}^{n}} \int_{\mathcal{S}}\left(W_{i}^{L}-W_{i}^{R}\right) \widehat{P}_{i}^{n c} d \mathcal{S} \\
& +\sum_{\mathcal{S} \in \mathcal{S}^{n}} \int_{\mathcal{S}}\left\{\left\{W_{i}\right\}\left(\int_{0}^{1} G_{i k r}\left(\phi\left(\tau ; U^{L}, U^{R}\right)\right) \frac{\partial \phi_{r}}{\partial \tau}\left(\tau ; U^{L}, U^{R}\right) d \tau \bar{n}_{k}^{L}\right) d \mathcal{S} .\right.
\end{aligned}
$$

The last term makes it different from standard discontinuous Galerkin finite element formulations. It is needed to introduce a measure for the nonconservative product where $U$ is discontinuous. Note that an extra function, $\phi\left(\tau ; U_{L}, U_{R}\right)$, has been introduced to deal with the regularization of $U$ across the discontinuity. In [27] the effect of the choice of $\phi\left(\tau ; U_{L}, U_{R}\right)$ on the numerical solution was investigated. We concluded that the numerical diffusion has a regularizing effect across discontinuities, which significantly reduces the dependence of the solution on $\phi\left(\tau ; U_{L}, U_{R}\right)$, so that often it does not matter in practice how $\phi\left(\tau ; U_{L}, U_{R}\right)$ is chosen. We adopt a linear path: $\phi\left(\tau ; U_{L}, U_{R}\right)=U_{L}+\tau\left(U_{R}-U_{L}\right)$. Furthermore, we use here the NCP numerical flux $\widehat{P}^{n c}\left(U^{L}, U^{R}, v, \bar{n}^{L}\right)$ designed in [27] for systems containing nonconservative products as a generalization of the HLL flux [31]. The NCP numerical flux $\widehat{P}^{n c}\left(U^{L}, U^{R}, v, \bar{n}^{L}\right)$ reads:

$\widehat{P}_{i}^{n c}\left(U_{L}, U_{R}, v, \bar{n}^{L}\right)=\left\{\begin{array}{c}F_{i k}^{L} \bar{n}_{k}^{L}-\frac{1}{2} \int_{0}^{1} G_{i k r}\left(\bar{\phi}\left(\tau ; U_{L}, U_{R}\right)\right) \frac{\partial \bar{\phi}_{r}}{\partial \tau}\left(\tau ; U_{L}, U_{R}\right) d \tau \bar{n}_{k}^{L} \\ \text { if } S_{L}>v, \\ \left\{F_{i k}\right\} \bar{n}_{k}^{L}+\frac{1}{2}\left(\left(S_{R}-v\right) \bar{U}_{i}^{*}+\left(S_{L}-v\right) \bar{U}_{i}^{*}-S_{L} U_{i}^{L}-S_{R} U_{i}^{R}\right) \\ \text { if } S_{L}<v<S_{R}, \\ F_{i k}^{R} \bar{n}_{k}^{L}+\frac{1}{2} \int_{0}^{1} G_{i k r}\left(\bar{\phi}\left(\tau ; U_{L}, U_{R}\right)\right) \frac{\partial \bar{\phi}_{r}}{\partial \tau}\left(\tau ; U_{L}, U_{R}\right) d \tau \bar{n}_{k}^{L} \\ \text { if } S_{R}<v,\end{array}\right.$ 
with $\bar{U}^{*}$ given by:

$$
\begin{aligned}
\bar{U}_{i}^{*}=\frac{S_{R} U_{i}^{R}-S_{L} U_{i}^{L}+\left(F_{i k}^{L}-F_{i k}^{R}\right) \bar{n}_{k}^{L}}{S_{R}-S_{L}}- \\
\frac{1}{S_{R}-S_{L}} \int_{0}^{1} G_{i k r}\left(\phi\left(\tau ; U_{L}, U_{R}\right)\right) \frac{\partial \phi_{r}}{\partial \tau}\left(\tau ; U_{L}, U_{R}\right) d \tau \bar{n}_{k}^{L} .
\end{aligned}
$$

Note that the first terms on the right hand side of (7) are in each case the upwind or unstable numerical fluxes. The wave speeds $S_{L}$ and $S_{R}$ in the numerical flux are usually approximated by the minimum and maximum eigenvalues of the Jacobian matrix. The characteristic polynomial of the Jacobian matrix of the depth-averaged model, $\partial F / \partial U+G$ is $c(\lambda)=\left(\lambda-q_{v}\right)\left(\lambda-q_{u}\right) p(\lambda)$ in which $p(\lambda)=\lambda^{4}+a_{1} \lambda^{3}+a_{2} \lambda^{2}+a_{3} \lambda+a_{4}$, where

$$
\begin{aligned}
a_{1}= & -2\left(q_{u}+q_{v}\right), \\
a_{2}= & q_{u}^{2}+q_{v}^{2}+4 q_{u} q_{v}-\varepsilon g_{3} h(1-\alpha+\rho \alpha), \\
& -\frac{1}{2} \varepsilon g_{3} h(1-\rho)(1+\alpha)\left(\varphi_{11} n_{1}^{2}+\varphi_{22} n_{2}^{2}+\varphi_{12} n_{1} n_{2}+\varphi_{21} n_{1} n_{2}\right) \\
a_{3}= & -2 q_{u} q_{v}\left(q_{u}+q_{v}\right)+2 q_{v} \varepsilon g_{3} h(1-\alpha)+2 \varepsilon \rho g_{3} \alpha h q_{u} \\
& +2 q_{u}\left(\frac{1}{2} \varepsilon g_{3} h(1+\alpha)(1-\rho)\left(\varphi_{11} n_{1}^{2}+\varphi_{22} n_{2}^{2}+\varphi_{12} n_{1} n_{2}+\varphi_{21} n_{1} n_{2}\right)\right), \\
a_{4}= & q_{u}^{2} q_{v}^{2}-q_{u}^{2}\left(\frac{1}{2} h \varepsilon g_{3}(1-\rho)(1+\alpha)\left(\varphi_{11} n_{1}^{2}+\varphi_{22} n_{2}^{2}+\varphi_{12} n_{1} n_{2}+\varphi_{21} n_{1} n_{2}\right)\right) \\
& +\frac{1}{2} \varepsilon^{2} g_{3}^{2} h^{2}(1-\rho)(1-\alpha)\left(\varphi_{11} n_{1}^{2}+\varphi_{22} n_{2}^{2}+\varphi_{12} n_{1} n_{2}+\varphi_{21} n_{1} n_{2}\right) \\
& -q_{v}^{2} \varepsilon g_{3} h(1-\alpha)-q_{u}^{2} \varepsilon \rho g_{3} \alpha h .
\end{aligned}
$$

Two eigenvalues are $\lambda_{1}=q_{v}$ and $\lambda_{2}=q_{u}$. Since explicitly solving the quartic polynomial $p(\lambda)=0$ yields rather unwieldy relations, we approximate the remaining four eigenvalues.

We approximate $p(\lambda)$ by $\tilde{p}(\lambda)=\left(\lambda-q^{u}-\mathcal{A}\right)\left(\lambda-q^{u}+\mathcal{A}\right)\left(\lambda-q^{v}-\mathcal{B}\right)\left(\lambda-q^{v}+\mathcal{B}\right)$ and expand $\tilde{p}$ as $\tilde{p}=\lambda^{4}+a_{1} \lambda^{3}+b_{2} \lambda^{2}+b_{3} \lambda+b_{4}$ with coefficients:

$$
\begin{aligned}
& b_{2}=q_{u}^{2}+q_{v}^{2}+4 q_{u} q_{v}-\left(\mathcal{A}^{2}+\mathcal{B}^{2}\right) \\
& b_{3}=2 q_{v} \mathcal{A}^{2}+2 q_{u} \mathcal{B}^{2}-2 q_{v} q_{u}\left(q_{u}+q_{v}\right), \\
& b_{4}=q_{v}^{2} q_{u}^{2}-q_{u}^{2} \mathcal{B}^{2}-q_{v}^{2} \mathcal{A}^{2}+\mathcal{A}^{2} \mathcal{B}^{2}
\end{aligned}
$$

Note that by choosing

$$
\begin{aligned}
& \mathcal{A}=\sqrt{\varepsilon g_{3} h(1-\alpha)}, \\
& \mathcal{B}=\sqrt{\frac{1}{2} h \varepsilon g_{3}(1-\rho)(1+\alpha)\left(\varphi_{11} n_{1}^{2}+\varphi_{22} n_{2}^{2}+\varphi_{12} n_{1} n_{2}+\varphi_{21} n_{1} n_{2}\right)},
\end{aligned}
$$

the coefficients $a_{i}$ and $b_{i}$ almost match. We approximate the solutions to $p(\lambda)$ now as $\lambda_{3,4}=q_{u} \pm \mathcal{A}$ and $\lambda_{5,6}=q_{v} \pm \mathcal{B}$. The error in the approximation of the roots is then proportional to $p\left(\lambda_{3,4}\right)=\mathcal{O}\left(\varepsilon^{2}\right)$ and $p\left(\lambda_{5,6}\right)=\mathcal{O}(\varepsilon)$.

As mentioned above, $\phi\left(\tau ; U_{L}, U_{R}\right)$ had to be chosen and we adopted $\phi\left(\tau ; U_{L}, U_{R}\right)=$ 
$U_{L}+\tau\left(U_{R}-U_{L}\right)$. This choice of the path presents us the opportunity to exactly determine the integral due to the nonconservative product in (6):

$$
\int_{0}^{1} G_{k r}\left(\phi\left(\tau ; U_{L}, U_{R}\right)\right) \frac{\partial \phi_{r}}{\partial \tau}\left(\tau ; U_{L}, U_{R}\right) d \tau \bar{n}_{k}^{L}=\left[\begin{array}{c}
0 \\
0 \\
-\varepsilon \rho g_{3} \llbracket h \rrbracket \int_{0}^{1} \alpha h d \tau \\
-\varepsilon \rho g_{3} \llbracket h \rrbracket \int_{0}^{1} \alpha h d \tau \\
-\varepsilon g_{3} \llbracket h \rrbracket \int_{0}^{1}(1-\alpha) h d \tau \\
-\varepsilon g_{3} \llbracket h \rrbracket \int_{0}^{1}(1-\alpha) h d \tau
\end{array}\right],
$$

in which

$$
\begin{aligned}
\int_{0}^{1} \alpha h d \tau & =\frac{1}{3}\left(\alpha_{L} h_{L}+\frac{1}{2}\left(\alpha_{R} h_{L}+\alpha_{L} h_{R}\right)+\alpha_{R} h_{R}\right), \\
\int_{0}^{1}(1-\alpha) h d \tau & =\left\{\{h\}-\frac{1}{3}\left(\alpha_{L} h_{L}+\frac{1}{2}\left(\alpha_{R} h_{L}+\alpha_{L} h_{R}\right)+\alpha_{R} h_{R}\right) .\right.
\end{aligned}
$$

\subsection{Pseudo-time stepping}

By replacing $U$ and $W$ in the weak formulation (6) by their polynomial expansions (5), a system of algebraic equations for the expansion coefficients of $U$ is obtained. For each physical time step, the system can be written as:

$$
\mathcal{L}\left(\hat{U}^{n} ; \hat{U}^{n-1}\right)=0 .
$$

This system of coupled non-linear equations is solved by adding a pseudo-time derivative of the primitive variables $V=\left[h, \alpha, v_{i}, u_{i}\right]^{T}$, hence (13) becomes:

$$
\mathcal{M} \frac{\partial \hat{V}^{n}}{\partial \tau}=-\mathcal{L}\left(\hat{V}^{n} ; \hat{V}^{n-1}\right), \quad \mathcal{M}=\int_{\mathcal{K}} \phi \frac{\partial U}{\partial V} d \mathcal{K},
$$

which is integrated to steady-state in pseudo-time. Following Van der Vegt and Van der Ven [32], we use the explicit Runge-Kutta method for inviscid flow with Melson correction given by:

Algorithm 1 Five-stage explicit Runge-Kutta scheme:

(1) Initialize $\hat{Y}^{0}=\hat{V}$.

(2) For all stages $s=1$ to 5 :

$$
\left(I+\alpha_{s} \lambda I\right) \hat{Y}^{s}=\hat{Y}^{0}+\alpha_{s} \lambda\left(\hat{Y}^{s-1}-\mathcal{M}^{-1} \mathcal{L}\left(\hat{Y}^{s-1} ; \hat{V}^{n-1}\right)\right) .
$$

(3) Update $\hat{V}=\hat{Y}^{5}$. 


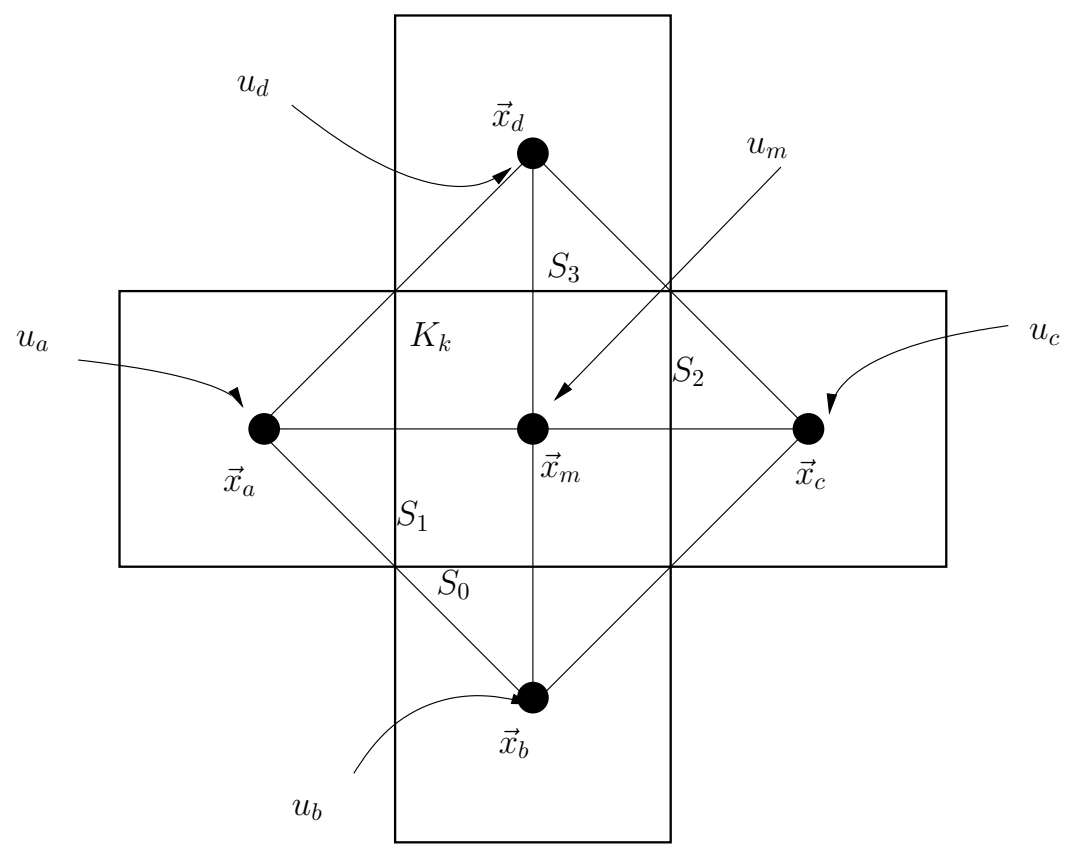

Fig. 5. Slope limiter in 2D.

The coefficient $\lambda$ is defined as $\lambda=\Delta \tau / \Delta t$, with $\Delta \tau$ the pseudo-time step and $\Delta t$ the physical time step. The Runge-Kutta coefficients $\alpha_{s}$ use are [32]: $\alpha_{1}=0.0797151, \alpha_{2}=0.163551, \alpha_{3}=0.283663, \alpha_{4}=0.5$ and $\alpha_{5}=1.0$.

\subsection{Slope limiter and discontinuity detector}

In numerical discretizations of the weak formulation (6), spurious oscillations generally appear near discontinuities. Using the Krivodonova discontinuity detector [17], we apply a slope limiter only near discontinuities to deal with these spurious oscillations. We use the slope limiter given in [21] which we describe briefly here for reasons of clarity.

The idea of the slope limiter is to replace the original polynomial $P_{0}$ by a new polynomial $P$ that uses the data $u_{m}$ of the midpoints of the original element in element $K_{k}$ and its neighboring elements $u_{a}, u_{b}, u_{c}$ and $u_{d}$. Eight polynomials are constructed, 4 Lagrange polynomials, $P_{i}, i=1,2,3,4$ and 4 Hermite polynomials $P_{i}, i=5,6,7,8$. For the Hermite polynomials we also need the physical gradient of the data in the neighboring elements at the points $\vec{x}$, i.e., $\nabla u_{a}, \nabla u_{b}, \nabla u_{c}$ and $\nabla u_{d}$ (see Fig. 5).

To construct the Lagrange polynomials consider the surface through $x_{m}, x_{a}$ and $x_{b}$. Name the polynomial through this surface $P_{1}$ with $P_{1}=\hat{P}_{1}^{a}+\hat{P}_{1}^{b} x+\hat{P}_{1}^{c} y$. 
The coefficients $\hat{P}_{1}^{a}, \hat{P}_{1}^{b}$ and $\hat{P}_{1}^{c}$ are found by solving:

$$
\left[\begin{array}{ccc}
1 & x_{m} & y_{m} \\
1 & x_{a} & y_{a} \\
1 & x_{b} & y_{b}
\end{array}\right]\left[\begin{array}{c}
\hat{P}_{1}^{a} \\
\hat{P}_{1}^{b} \\
\hat{P}_{1}^{c}
\end{array}\right]=\left[\begin{array}{l}
u_{m} \\
u_{a} \\
u_{b}
\end{array}\right]
$$

In the same way, polynomials $P_{2}, P_{3}$ and $P_{4}$ are constructed by considering the remaining three surfaces.

Each of the four Hermite polynomials are determined by looking at the current element and one of the neighbors, e.g., the first Hermite polynomial, $P_{5}$, is found by looking at the neighboring element sharing face $S_{0}$. In the midpoint $x_{b}$, the gradient of the solution is $\nabla u_{b}$, while the solution in the midpoint of the current element is $u_{m}$. The first Hermite polynomial is given by: $P_{5}=\hat{P}_{5}^{a}+\hat{P}_{5}^{b} x+\hat{P}_{5}^{c} y$ where:

$$
\begin{aligned}
& \hat{P}_{5}^{a}=u_{m}-x_{b} \cdot \nabla u_{b}, \\
& \hat{P}_{5}^{b}=\partial_{x} u_{b} \quad \text { in } x_{b}, \\
& \hat{P}_{5}^{c}=\partial_{y} u_{b} \quad \text { in } x_{b} .
\end{aligned}
$$

In the same way, polynomials $P_{6}, P_{7}$ and $P_{8}$ are constructed by considering the remaining three surfaces.

The linear approximation of the original polynomial is determined just like the Hermite polynomials. In the midpoint $x_{m}$, the solution is $u_{m}$ and the gradient is $\nabla u_{m}$. The linear approximation is: $P_{0}=\hat{P}_{0}^{a}+\hat{P}_{0}^{b} x+\hat{P}_{0}^{c} y$ where:

$$
\begin{aligned}
& \hat{P}_{0}^{a}=u_{m}-x_{m} \cdot \nabla u_{m}, \\
& \hat{P}_{0}^{b}=\partial_{x} u_{m} \quad \text { in } x_{m}, \\
& \hat{P}_{0}^{c}=\partial_{y} u_{m} \quad \text { in } x_{m} .
\end{aligned}
$$

Now project $P_{j}, j=0, \ldots, 8$, onto the DG space and solve for $\left(\hat{u}_{0}\right)_{j},\left(\hat{u}_{1}\right)_{j}$ and $\left(\hat{u}_{2}\right)_{j}$ :

$$
\left[\begin{array}{ccc}
\int_{K_{k}} \psi_{0} \psi_{0} d K & \int_{K_{k}} \psi_{0} \psi_{1} d K & \int_{K_{k}} \psi_{0} \psi_{2} d K \\
\int_{K_{k}} \psi_{1} \psi_{0} d K & \int_{K_{k}} \psi_{1} \psi_{1} d K & \int_{K_{k}} \psi_{1} \psi_{2} d K \\
\int_{K_{k}} \psi_{2} \psi_{0} d K & \int_{K_{k}} \psi_{2} \psi_{1} d K & \int_{K_{k}} \psi_{2} \psi_{2} d K
\end{array}\right]\left[\begin{array}{l}
\left(\hat{u}_{0}\right)_{j} \\
\left(\hat{u}_{1}\right)_{j} \\
\left(\hat{u}_{2}\right)_{j}
\end{array}\right]=\left[\begin{array}{l}
\int_{K_{k}} \psi_{0} P_{j} d K \\
\int_{K_{k}} \psi_{1} P_{j} d K \\
\int_{K_{k}} \psi_{2} P_{j} d K
\end{array}\right] .
$$

After the polynomial reconstruction is performed, an oscillation indicator is used to assess the smoothness of $P_{i}$. The oscillation indicator for the polynomial $P_{i}, i=0, \ldots, 8$, is defined as $o_{i}=\left\|\nabla P_{i}\right\|$, with $\|\cdot\|$ the Euclidian norm. The coefficients of the new solution $u$ in element $K_{k}$ are constructed as the sum of 
all the polynomials multiplied by a weight, $\hat{u}_{q}=\sum_{i=0}^{8} w_{i}\left(\hat{u}_{q}\right)_{i}, q=0,1,2$, in which the weights are computed as:

$$
w_{i}=\frac{\left(\epsilon+o_{i}\left(P_{i}\right)\right)^{-\gamma}}{\sum_{j=0}^{8}\left(\epsilon+o_{i}\left(P_{j}\right)\right)^{-\gamma}},
$$

where $\gamma$ is a positive number and $\epsilon$ a small number to avoid division by 0 . Take for example $\epsilon=10^{-12}$. The effect of $\gamma$ and the combination of polynomials (Lagrange and original or Lagrange, original and Hermite) is tested in Section 4.2.

The discontinuity detector introduced in Krivodonova et al. [17] defines for each element $\mathcal{K}_{k}^{n}$ a measure of the discontinuity $\mathcal{I}_{k}$. This will indicate regions where the gradient of a variable $\mathcal{V}$ is large. For the depth-averaged two-phase flow equations, depending on the situation, we choose either $\mathcal{V}=h$ and $\mathcal{V}=\alpha$. The discontinuity detector is given by:

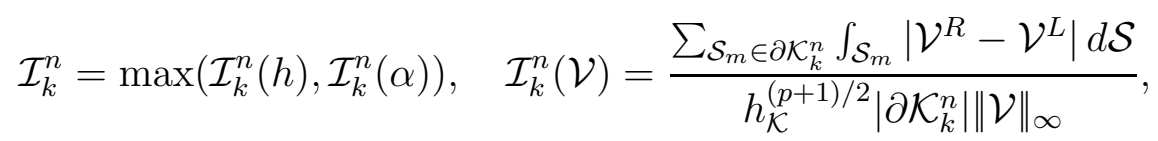

where $h_{\mathcal{K}}$ is the cell measure defined as the radius of the largest circumscribed circle in the element $\mathcal{K}_{k}^{n}, p$ the polynomial order, $\left|\partial \mathcal{K}_{k}^{n}\right|$ the surface area of the element and $\|\cdot\|_{\infty}$ the maximum norm. The solution is estimated [17] to be smooth when $\mathcal{I}_{k}<1$ and non-smooth when $\mathcal{I}_{k}>1$.

\section{Verification}

\subsection{Sub- and supercritical flow over a bump}

We consider the 1D steady-state solution of sub- and supercritical flow over a bump [27]. This is a popular test case to verify shallow water codes $[5,12,19,35,30]$ and we extend the test case to the depth-averaged two-phase flow model. For this test case we consider:

$$
\left[\begin{array}{c}
h(1-\alpha) \\
h \alpha \\
h \alpha v \\
h(1-\alpha) u \\
b
\end{array}\right]_{t}\left[\begin{array}{c}
h(1-\alpha) u \\
h \alpha v \\
h \alpha v^{2}+\frac{1}{2} \varepsilon(1-\rho) \varphi_{11} g_{3} h^{2} \alpha \\
h(1-\alpha) u^{2} \\
0
\end{array}\right]_{x}+\left[\begin{array}{ccccc}
0 & 0 & 0 & 0 & 0 \\
0 & 0 & 0 & 0 & 0 \\
G_{31} & 0 & 0 & 0 & G_{35} \\
G_{41} & 0 & 0 & 0 & G_{45} \\
0 & 0 & 0 & 0 & 0
\end{array}\right]\left[\begin{array}{l}
h \\
\alpha \\
v \\
u \\
b
\end{array}\right]_{x}=\left[\begin{array}{c}
0 \\
0 \\
S_{3} \\
S_{4} \\
0
\end{array}\right],
$$


where

$$
\begin{aligned}
G_{31} & =\varepsilon \rho \alpha g_{3} h, \quad G_{35}=\varepsilon(1-\rho) \varphi_{11} g_{3} h \alpha+\varepsilon \rho h \alpha g_{3} \\
G_{41} & =\varepsilon(1-\alpha) g_{3} h, \quad G_{45}=\varepsilon(1-\alpha) g_{3} h \\
S_{3} & =h F^{D}, \quad S_{4}=-h F^{D} / \rho .
\end{aligned}
$$

Note that we take the given topography to be formally unknown in the system. This leads to a well-balanced scheme [27]. Let the upstream variables be denoted as $h_{0}, \alpha_{0}, u_{0}$ and $v_{0}$. For both the subcritical and supercritical test case we take $h_{0}=1, u_{0}=1, v_{0}=1$, and $\alpha=0.3$.

We consider the solution on a domain $x \in[0,1]$ in which the topography is given by [35]:

$$
b(x)= \begin{cases}0.2-20(x-0.5)^{2} & \text { if } 0.4 \leq x \leq 0.6 \\ 0 & \text { otherwise }\end{cases}
$$

As initial condition we take $h+b=1, u=u_{0}, v=v_{0}$ and $\alpha=\alpha_{0}$. At the boundaries we define the exterior trace to be the same as the initial condition. For the subcritical test case we take $g_{3}=10^{8}$ while for the supercritical test case $g_{3}=25$. Other parameters in the model are chosen as: $\varepsilon=0.01$, $\left.\rho=0.9, \theta=0^{\circ}, \varphi_{11}=2\left(1-\sqrt{1-\cos ^{2}\left(\phi_{\text {int }}\right)\left(1+\tan ^{2}\left(\phi_{\text {bed }}\right)\right.}\right)\right) / \cos ^{2}\left(\phi_{\text {int }}\right)-1$, $\phi_{\text {int }}=24.5^{\circ}$ and $\phi_{\text {bed }}=14.75^{\circ}$. In $F^{D}$, the parameters are $\rho^{f}=1000 \mathrm{~kg} \mathrm{~m}^{-3}$, $v_{T}=0.143 \mathrm{~m} \mathrm{~s}^{-1}, d=10^{-3} \mathrm{~m}$ and $\mu^{f}=10^{-3} \mathrm{~kg}(\mathrm{~ms})^{-1}$.

We compute the order of convergence by comparing the space-time discontinuous Galerkin finite element solution of (17) to an "exact" solution of (17). This "exact" solution is found by setting the time-derivative terms in (17) to zero and then solving the system of ODE's with a RK45 method on a grid with 10000 points.

In Figure 6 we plot the numerical solutions of the total flow height $h+b$, topography $b$, flow depth $h$, particle volume fraction $\alpha$ and the velocities $u$ and $v$ for sub- and supercritical flow. The order of convergence is given for the mixture momentum $h \alpha v+\rho h(1-\alpha) u$ as well as the topography $b$ in Table 1 for sub- and supercritical flow. The reason why we also show the order of convergence for the topography $b$ is because it is taken formally as an unknown in the system (as in [27]) and we show that the topography converges at the same rate as the other unknowns. For a linear polynomial approximation we obtain as expected second order convergence. 


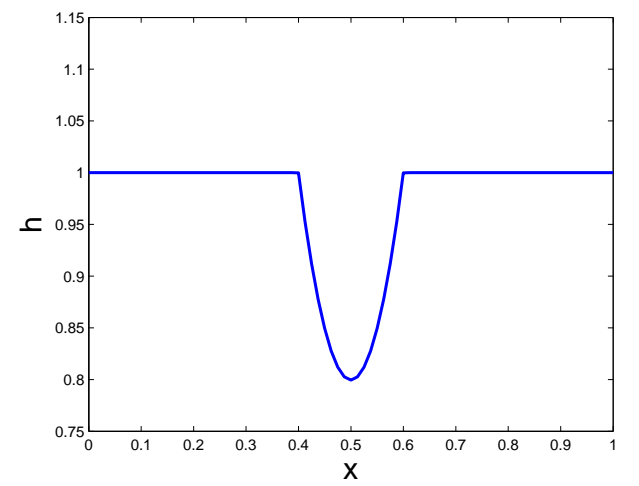

(a) Subcritical flow: flow depth $h$.

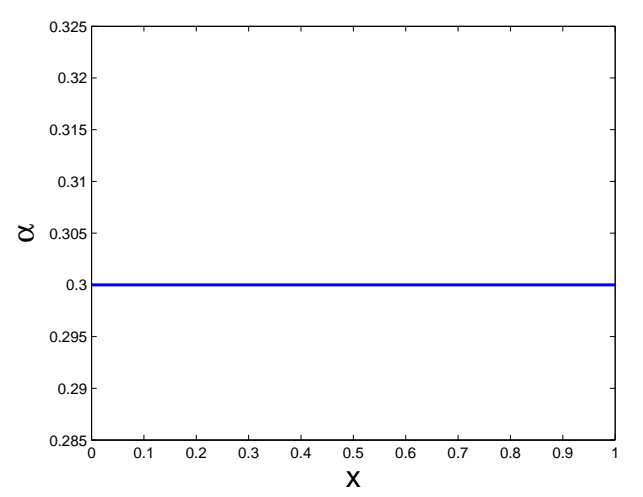

(c) Subcritical flow: particle volume frac- (d) Supercritical flow: particle volume tion $\alpha$.

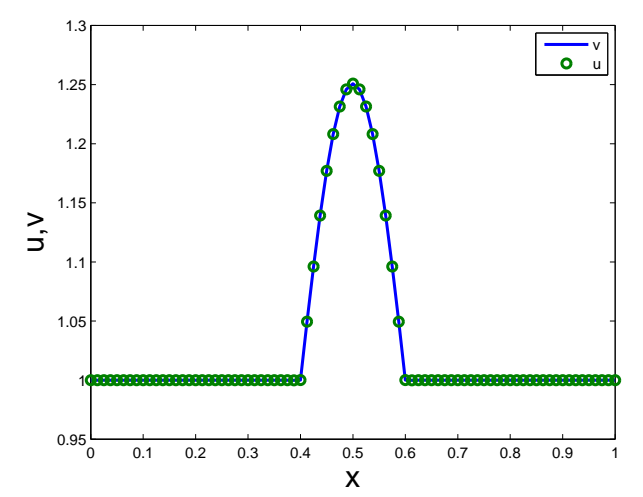

(e) Subcritical flow: velocities $u$ and $v$.

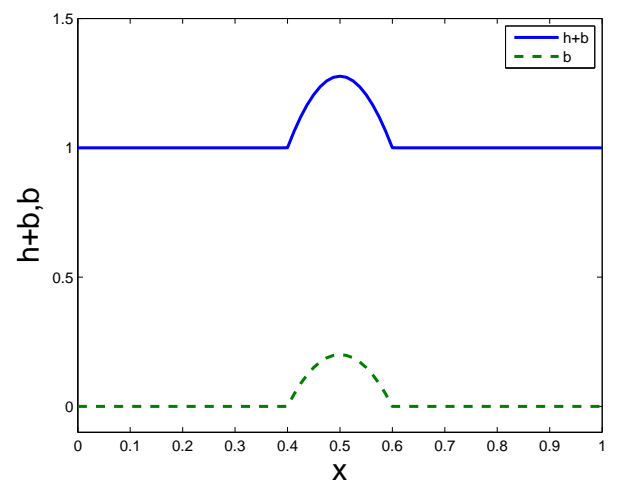

(b) Supercritical flow: flow height $h+b$ and topography $b$.

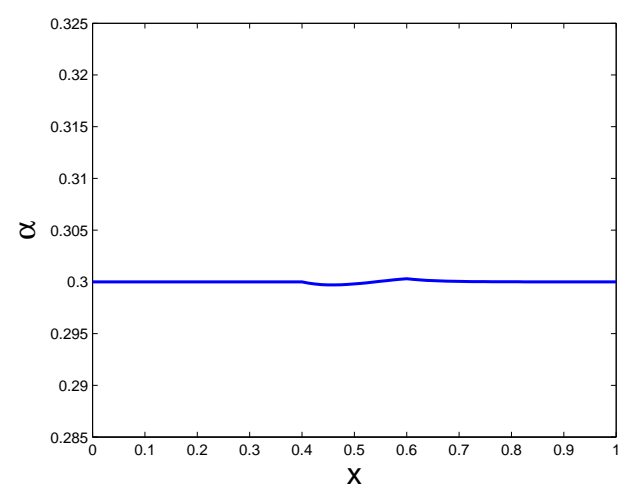

Fig. 6. Steady-state solution on a grid with 80 elements.

\subsection{The slope limiter}

We consider a Riemann problem to test the effect of the polynomials (Lagrange, original and/or Hermite) in the slope limiter and the parameter $\gamma$ (see 


\begin{tabular}{|c|c|c|c|c|c|c|c|c|}
\hline & & Sub & itical flow & & & Supe & ritical flow & \\
\hline$N_{\text {cells }}$ & $b$ & $p$ & $h(\alpha v+\rho(1-\alpha) u)$ & $p$ & $b$ & $p$ & $h(\alpha v+\rho(1-\alpha) u)$ & $p$ \\
\hline 10 & $1.5171 \cdot 10^{-2}$ & - & $4.2299 \cdot 10^{-3}$ & - & $1.2910 \cdot 10^{-2}$ & - & $4.0446 \cdot 10^{-4}$ & - \\
\hline 20 & $3.7397 \cdot 10^{-3}$ & 2.0 & $1.0484 \cdot 10^{-3}$ & 2.0 & $3.4861 \cdot 10^{-3}$ & 1.9 & $1.4343 \cdot 10^{-4}$ & 1.5 \\
\hline 40 & $9.3222 \cdot 10^{-4}$ & 2.0 & $2.9977 \cdot 10^{-4}$ & 1.8 & $9.0211 \cdot 10^{-4}$ & 2.0 & $3.7399 \cdot 10^{-5}$ & 1.9 \\
\hline 80 & $2.3296 \cdot 10^{-4}$ & 2.0 & $8.1480 \cdot 10^{-5}$ & 1.9 & $2.2925 \cdot 10^{-4}$ & 2.0 & $9.4394 \cdot 10^{-6}$ & 2.0 \\
\hline
\end{tabular}

Table 1

Sub- and supercritical flow: $L^{2}$ error for the topography $b$ and the total momentum $h(\alpha v+\rho(1-\alpha) u)$ and the convergence rate $p$.

(15)) in the space-time DGFEM discretization. For this test case we neglect the source terms. Furthermore, we simplify the expressions for $\varphi_{11}, \varphi_{22}, \varphi_{12}$ and $\varphi_{21}$ by taking $\varphi_{11}=\varphi_{22}=1$ and $\varphi_{12}=\varphi_{21}=0$. Other parameters in the model are chosen as $\rho=1, g=1, \varepsilon=1$ and $\theta=0^{\circ}$. We consider the solution on a domain $[0,1] \times[0,1]$ divided into $32 \times 32$ elements. A physical time step of $\Delta t=0.005$ is used and we consider the solution at final time $T=0.37$. We consider the following two-dimensional Riemann problem:

$$
V(t=0)= \begin{cases}V^{L} & \text { for } x<0.5 \text { and } y<0.5 \\ V^{R} & \text { otherwise }\end{cases}
$$

in which $V$ is the vector of primitive variables and $V^{L}=\left[\begin{array}{ll}1,0.4,0,0,0,0 & 0\end{array}\right]^{T}$ and $V^{R}=[0.5,0.2,0,0,0,0]^{T}$. At the boundaries we set $u_{1}=u_{2}=v_{1}=$ $v_{2}=0$.

The slope limiter is used in element $\mathcal{K}_{k}^{n}$ only when the discontinuity detector $\mathcal{I}_{k}^{n}>\varepsilon_{k r i v}$. In this test case we take $\varepsilon_{k r i v}=1$.

In Figure 7 we compare the solution of the volume fraction $\alpha$ along the diagonal $x=y$ as computed using a slope limiter with the combination Lagrange and original polynomials; and, the combination Lagrange, Hermite and original polynomials. For each combination we furthermore compare the solution using $\gamma=1$ and $\gamma=10$ in (15). We see that the least numerical dissipation is introduced using the combination Lagrange and original polynomials while $\gamma=1$. Increasing $\gamma$ to $\gamma=10$ introduces more smoothing to the solution. Also adding the Hermite polynomials to the combination Lagrange and original polynomials increases the amount of numerical dissipation. This can also be seen in Figure 8 where we compare the flow height $h$ calculated using the combination Lagrange and original polynomials with $\gamma=1$; and, the combination Lagrange, Hermite and original polynomials with $\gamma=10$. We plot the results per element to show the discontinuities at the element faces which would not 


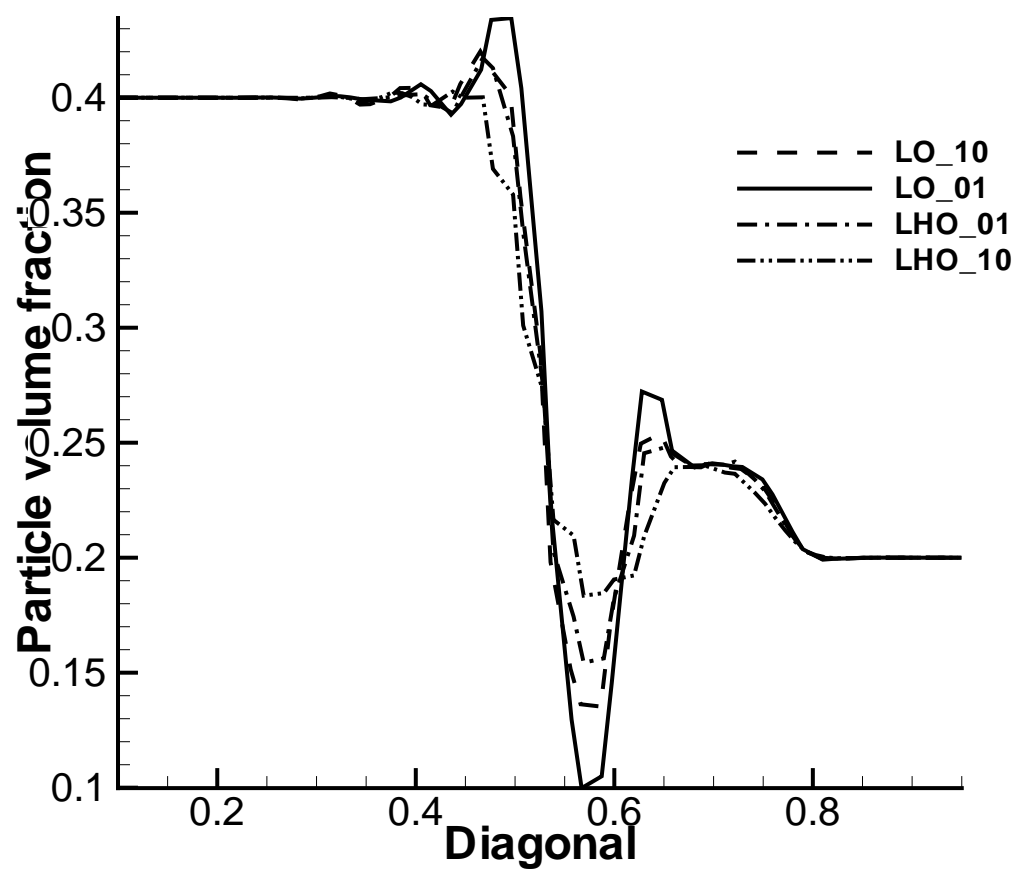

Fig. 7. Solution of the volume fraction $\alpha$ at $T=0.37$ along the diagonal $x=y$ as computed using the combination of Lagrange and original polynomials (LO) in the slope limiter or the combination of Lagrange, Hermite and original polynomials (LHO) with $\gamma=1$ or $\gamma=10$.

be visible with post-processing. We remark that without the slope limiter it was not possible to do this test case because $\alpha$ became less than zero in regions around large discontinuities due to undershoots. In Figure 9 we indicate the areas where the discontinuity detector detects large discontinuities. In these regions the slope limiter is used. The scheme is robust for a wide range of $\gamma$ values, but for accuracy reasons $\gamma$ should be chosen as small as possible, because this minimizes the numerical dissipation. For the Riemann problem presented here, the best combination would be the Lagrange and original polynomials with $\gamma=1$. As can be seen in Figures 7 and 8 there is a wave crest which can be captured using the combination Lagrange and original polynomials with $\gamma=1$ which is not captured using the combination Lagrange, Hermite and original polynomials with $\gamma=10$ since this combination introduces too much numerical dissipation. For other applications though, more numerical dissiaption may be desirable to avoid large over- and undershoots which can be achieved by slightly increasing the value for $\gamma$ and/or using a different combination of polynomials. 


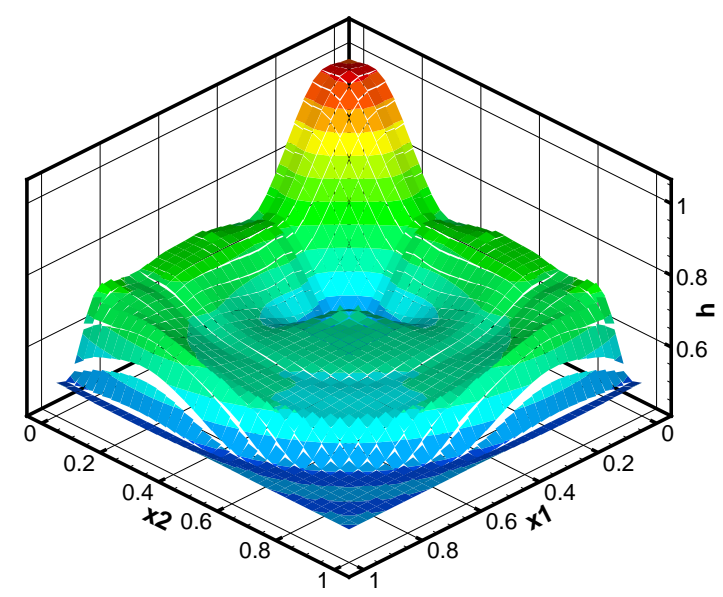

(a) The flow height $h$ as calculated using Lagrange and the original polynomials in the slope limiter. Furthermore, $\gamma=1$ in (15).

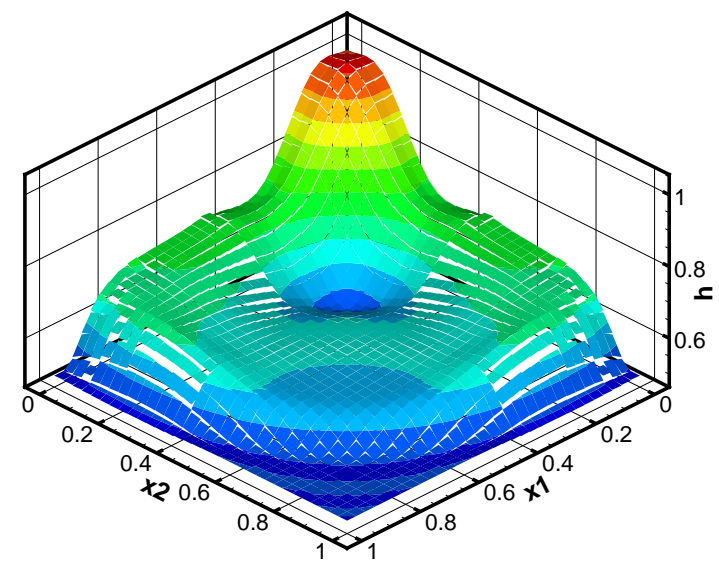

(b) The flow height $h$ as calculated using Lagrange, Hermite and the original polynomials in the slope limiter. Furthermore, $\gamma=10$ in (15).

Fig. 8. Solution of the flow height $h$ at $T=0.37$. Too much numerical dissipation is introduced in (b) since there is a wave crest in (a) which is not captured in (b). 


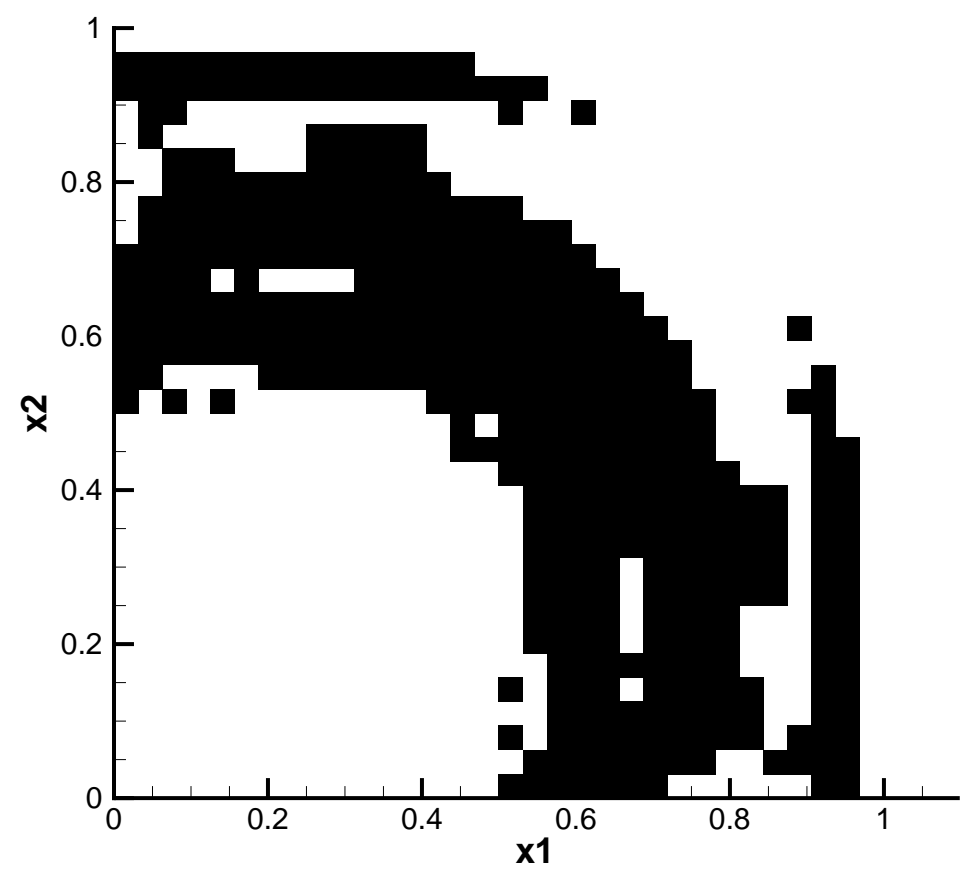

Fig. 9. The shaded area indicates where the discontinuity detector has large values and where the slope limiter is used; situation at $T=0.37$.

\section{Validation}

In $[1,2,30]$ laboratory experiments of shallow water and in [33] laboratory experiments of shallow granular flow through a contraction were compared to numerical results. We will simulate a two-phase flow mixture consisting of solid particles in water in which the density of the solid particles is slightly higher than that of water. We will simulate the flow of this mixture as it enters a contraction. Initially we start with a flow with very low particle volume fraction $(5 \%)$ and the flow reaches a steady-state with oblique jumps. We then perturb this steady-state by increasing the particle volume fraction at the inlet to $30 \%$ for a short period. This perturbation was sufficient to perturb the flow with oblique jumps to one with an upstream moving shock as was observed by Akers and Bokhove [1] (see Figure 2). We now describe the numerical setup.

In our numerical calculations we consider a channel in the cartesian coordinate system $(x, y) \in[0,10] \times[-0.5,0.5]$. The channel converges from $x=4$ to $x=4.7228$ so that $y \in[-0.3,0.3]$ and diverges from $x=4.7228$ to $x=6.1685$ (see Figure 10). As initial condition we take $h=0.2, \alpha=0.05, v_{1}=u_{1}=0.5$ and $v_{2}=u_{2}=0$. Define $h_{w}=h(1-\alpha)$. At the inflow boundary we specify $h_{w}=0.19$, the $x$-components of the velocities, $u_{1}=0.5$ and $v_{1}=0.5$, the $y$-components of the velocities, $u_{2}=0$ and $v_{2}=0$, and the particle volume 


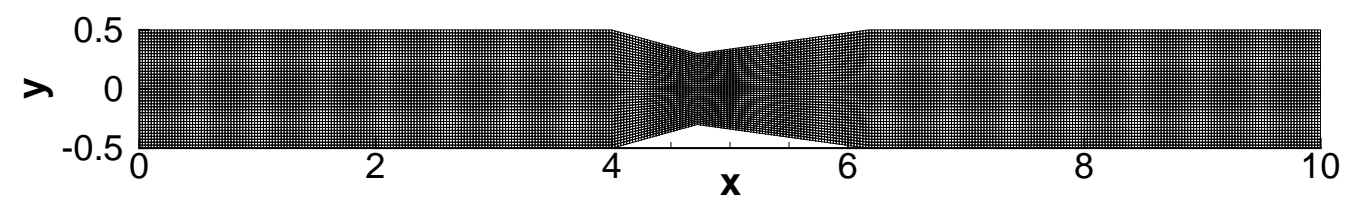

Fig. 10. Geometry and mesh of the chute with a contraction.

fraction $\alpha$. Initially, the inflow condition for the particle volume fraction is $\alpha=0.05$. For time $20<t<35$ we change the inflow condition by increasing the particle volume fraction to $\alpha=0.3$ after which we decrease the particle volume fraction again to $\alpha=0.05$. At the walls we follow Ambati and Bokhove [3] and impose:

$$
\begin{array}{ll}
\mathbf{v}^{b} \cdot \bar{n}=-\mathbf{v}^{L} \cdot \bar{n}, & \mathbf{v}^{b} \cdot \bar{t}=\mathbf{v}^{L} \cdot \bar{t} \\
\mathbf{u}^{b} \cdot \bar{n}=-\mathbf{u}^{L} \cdot \bar{n}, & \mathbf{u}^{b} \cdot \bar{t}=\mathbf{u}^{L} \cdot \bar{t}
\end{array}
$$

where $\bar{t}$ is the unit tangential vector orthogonal to the normal vector $\bar{n}$. Furthermore, we extrapolate the void fraction, $\alpha^{R}=\alpha^{L}$ and the flow height $h^{R}=h^{L}$. At the outflow boundary, all variables are extrapolated, $U^{R}=U^{L}$.

There are a number of constants in the depth-averaged flow model. We will consider shallow liquid-solid flows with a height to length ratio of $\varepsilon=0.2$ as a feasible approximation and for which the liquid to solid density ratio is $\rho=0.9$. The gravity constant is $g=1.5$ so that the gravity components are $g_{1}=\sin (\theta) g, g_{2}=0$ and $g_{3}=\cos (\theta) g$ in which $\theta$ is the angle of the contraction with respect to the horizontal (see also Figure 3). We take $\theta=0.625^{\circ}$ for $0 \leq x \leq 7$ and $\theta=10^{\circ}$ for $x>7$ so that the outflow boundary has no effect on the solution in the contraction. To be able to calculate the drag function $F^{D}$, we use the following constants: $\rho^{f}=1000 \mathrm{~kg} \mathrm{~m}^{-3}$ and $\mu^{f}=10^{-3} \mathrm{~kg}(\mathrm{~ms})^{-1}$ while the solid particles are assumed to have a diameter of $d=10^{-3} \mathrm{~m}$ and $v_{T}=0.143 \mathrm{~m} \mathrm{~s}^{-1}[15]$. The internal angle of friction is taken to be $\phi_{\text {int }}=24.5^{\circ}$ and the bed friction angle is $\phi_{\text {bed }}=14.75^{\circ}$ [4]. The bottom topography is taken constant $b(x, y)=0$ and the drag coefficient is $C_{D}=10^{-4}$.

We compute the solution for the depth-averaged model using space DGFEM until $t=100$ using a CFL number of CFL $=0.8$ on a grid with 400 elements in the $x$-direction and 40 elements in the $y$-direction. In the slope limiter, a combination of Lagrange, Hermite and original polynomials was used with $\gamma=10$ to avoid severe over- and undershoots. In Figures 11- 13 we show the transi- 
tion of the flow height $h$ from oblique jumps to an upstream steady shock (for a comparison, see also Figure 2). We see the same as observed by Akers and Bokhove [1] that after perturbing the steady-state solution of oblique jumps, an upstream steady shock appears. We remark that if $C_{D}=0$, we do not get an upstream steady shock. As expected, an upstream moving shock appears.

\section{Conclusions}

Recently, a depth-averaged two-phase flow model was introduced by Pitman and Le [25] and Le [18] to model shallow debris flows. We slightly extended this model by including extra friction terms to simulate turbulent friction. The depth-averaged model contains nonconservative products which makes it numerically challenging to solve. In Rhebergen et al. [27] we developed a discontinuous Galerkin finite element method to deal with nonconservative products which we applied in this article to solve the depth-averaged twophase flow model of Le [18].

The DGFEM discretizations for the depth-averaged model was verified against steady-state flow solutions over a bump and we obtained second order convergence when using linear polynomial approximations. To prevent numerical oscillations, the WENO slope limiter [21] in combination with Krivodonova's discontinuity detector [17] was successfully applied. A Riemann problem solution was shown which could not be solved without the slope limiter due to severe undershoots.

Furthermore, the effect of the choice of the polynomials and the parameter $\gamma$ in the slope limiter were shown. The scheme is robust for a wide range of $\gamma$ values, but for accuracy reasons $\gamma$ should be chosen as small as possible, because this minimizes the numerical dissipation. Also adding the Hermite polynomials to the combination Lagrange and original polynomials increases the amount of numerical dissipation. This could be seen in the Riemann problem we investigated where there was a wave crest that could only be captured using the Lagrange and original polynomials and setting $\gamma=1$. Certain applications with strong gradients however need more numerical dissipation to avoid over- and undershoots so that $\gamma$ may need to be slightly increased. This was necessary e.g. in the validation test case where we used the combination of Lagrange, Hermite and original polynomials with $\gamma=10$.

Finally, we qualitatively validated the model by showing the ability of the model to capture the phenomenon in which a steady state solution with oblique jumps is perturbed, by an increase of particles for a short period in time, 


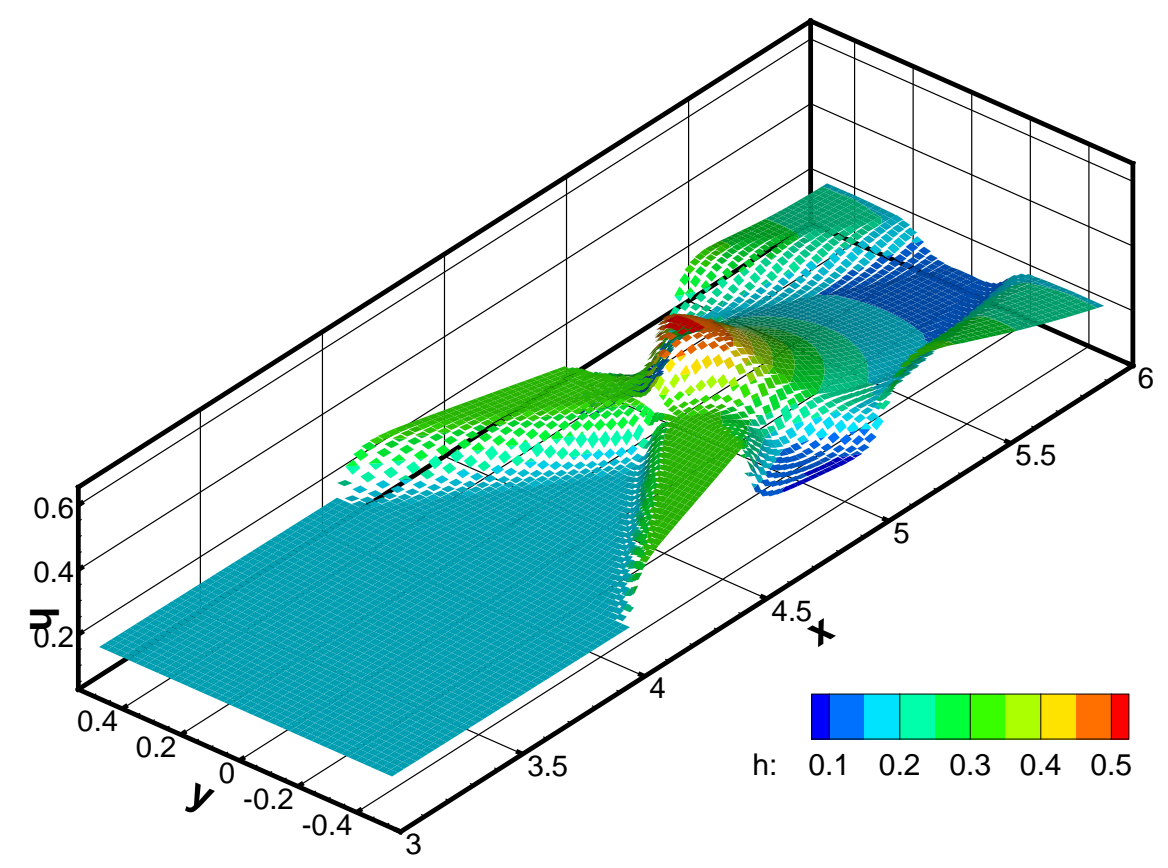

(a) Side view of $h$.
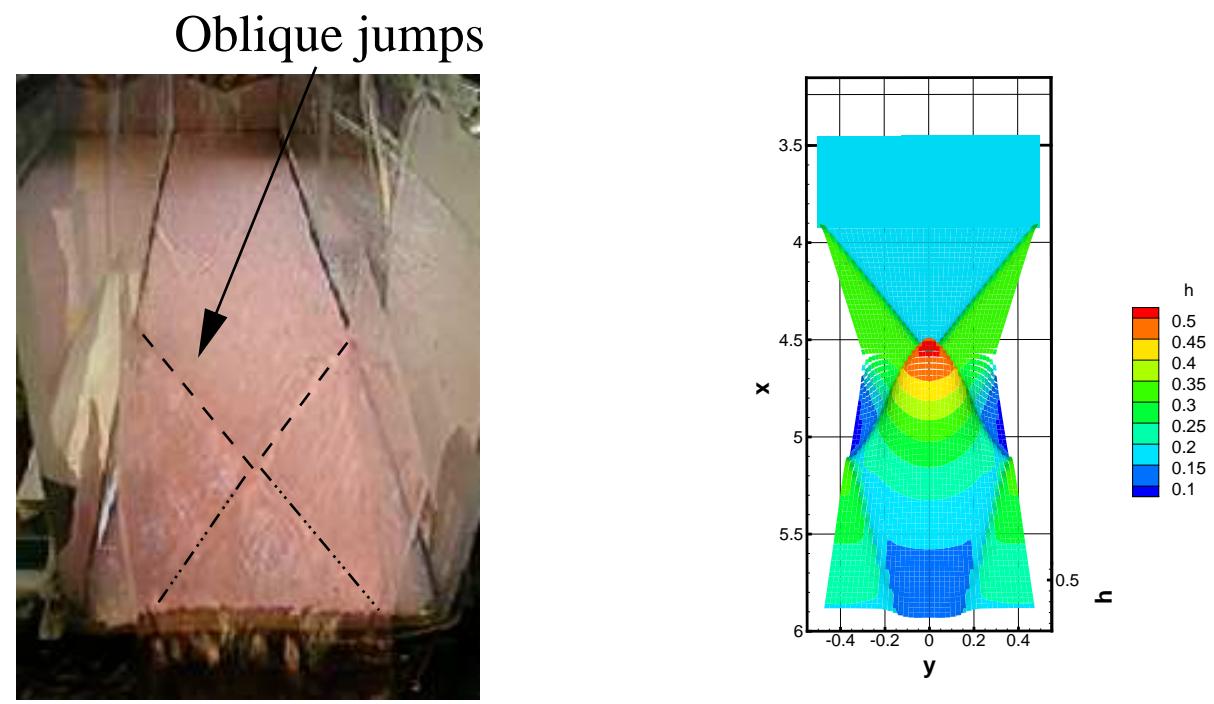

(b) Snapshot from the laboratory experiment.

(c) Top view of $h$.

Fig. 11. Oblique jump solution at $t=22$. 


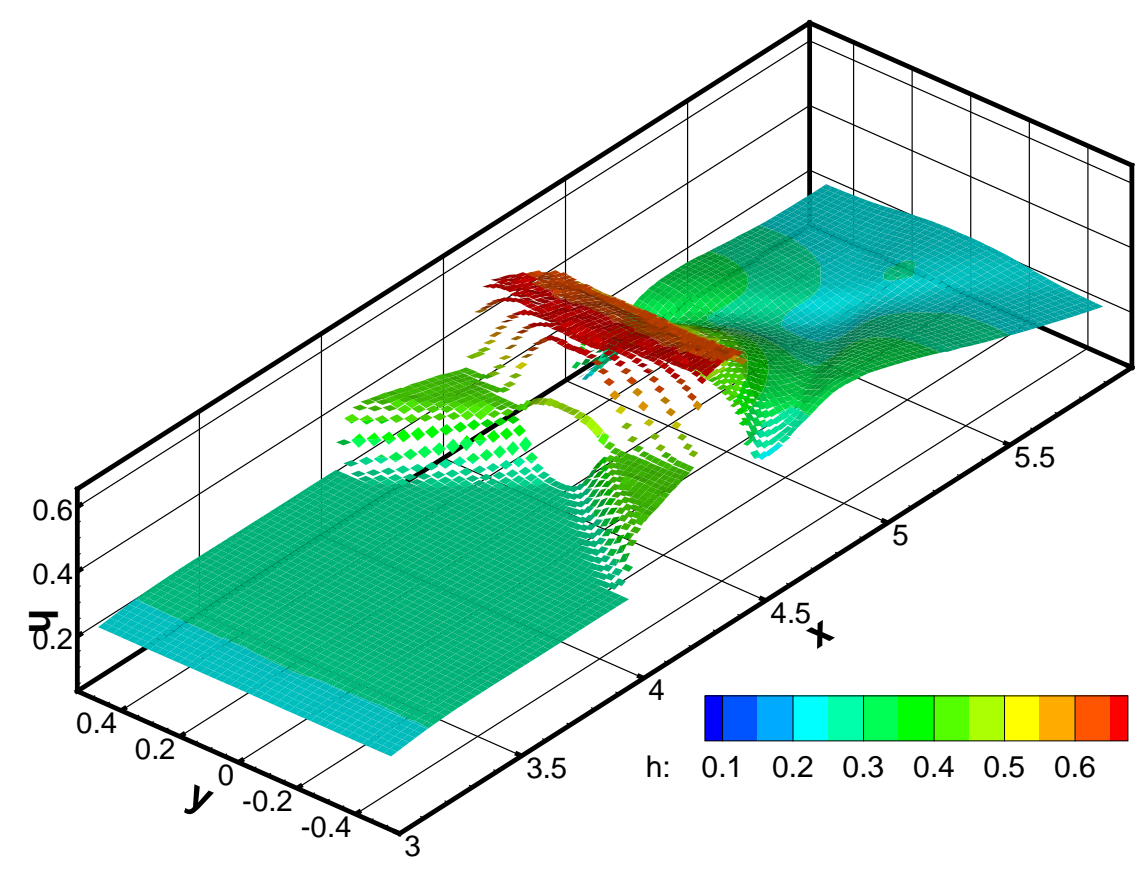

(a) Side view.

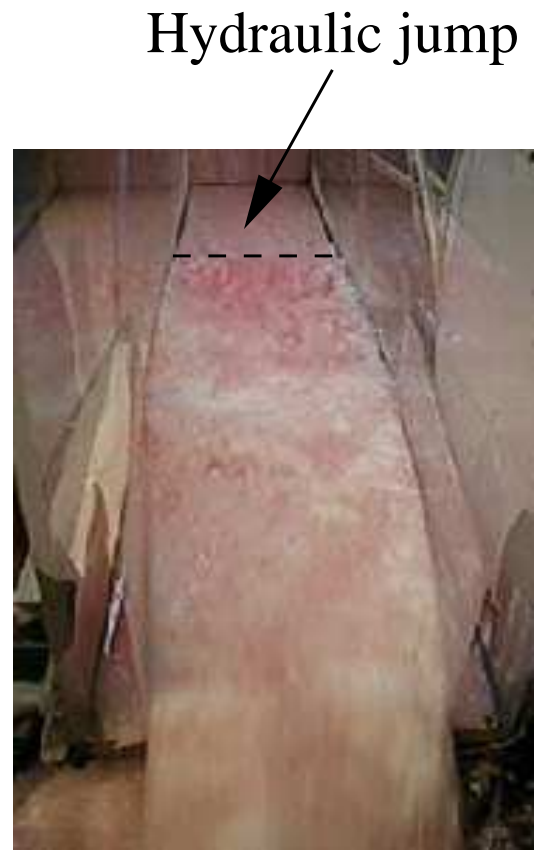

(b) Snapshot from the laboratory experiment.

Fig. 12. Transition phase at $t=39$. 


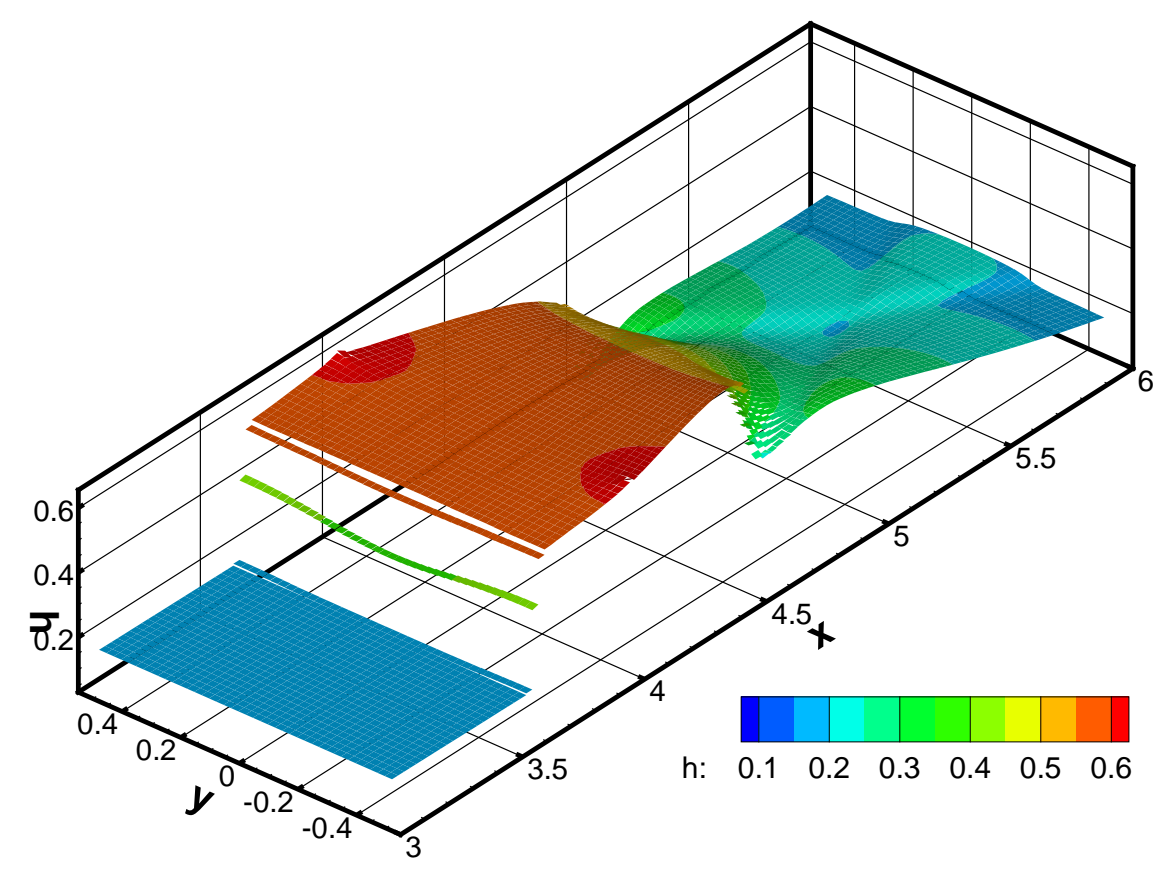

(a) Side view.

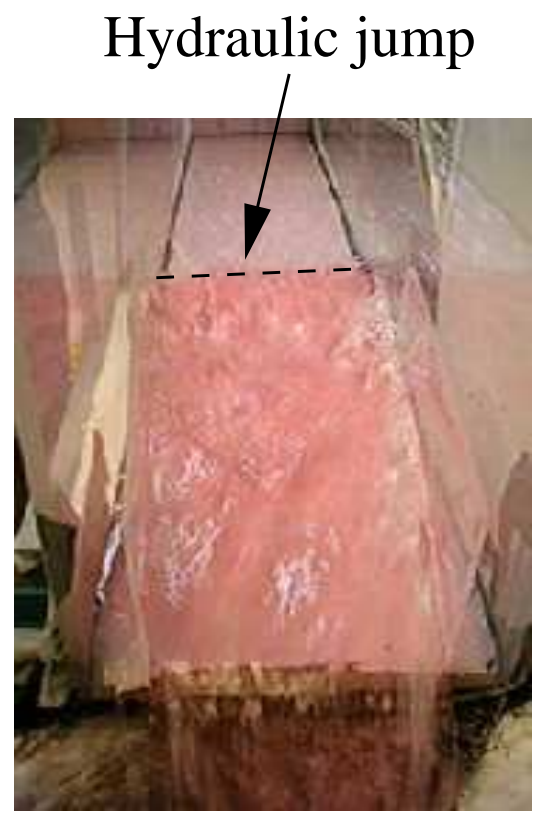

(b) Snapshot from the laboratory experiment.

Fig. 13. Upstream moving shock at $t=100$. 
so that an upstream steady shock appeared, as was observed by Akers and Bokhove [1].

\section{Acknowledgments}

S.R. and O.B. acknowledge support from the Institute of Mechanics, Processes and Control Twente. For J.V. this research was partly funded by the ICT project BRICKS (http://www.bsik-bricks.nl), theme MSV1. Furthermore, we would like to thank Henk Sollie for discussions on the slope limiter and Vijaya Ambati for proofreading.

\section{A The three-dimensional two-phase flow model}

In this section we present the three-dimensional two-phase flow model as derived by Jackson [15]. By depth-averaging this model, Pitman and Le [25] and Le [18] derived a depth-averaged two-phase flow model for shallow two-phase flows.

Assume that the only fluids stress is the fluids pressure. Furthermore, the densities $\rho^{f}$ and $\rho^{s}$ of both phases are assumed to be constant. The threedimensional model consists of two continuity equations and two momentum equations. To write the equations in compact form, we use the summation convention on repeated indices and the comma notation to denote partial differentiation. The continuity equations are given by:

$$
\begin{aligned}
\partial_{t}((1-\alpha))+\partial_{k}\left((1-\alpha) u_{k}\right) & =0 \\
\partial_{t}(\alpha)+\partial_{k}\left(\alpha v_{k}\right) & =0
\end{aligned}
$$

and the momentum equations as:

$$
\begin{aligned}
\partial_{t}\left((1-\alpha) \rho^{f} u_{i}\right)+\partial_{k}\left((1-\alpha) \rho^{f} u_{i} u_{k}\right) & =-(1-\alpha) \partial_{k}\left(\delta_{i k} p^{f}\right)-F_{i}^{D}+(1-\alpha) \rho^{f} g_{i}, \\
\partial_{t}\left(\alpha \rho^{s} v_{i}\right)+\partial_{k}\left(\alpha \rho^{s} v_{i} v_{k}+T_{i k}^{s}\right) & =-\alpha \partial_{k}\left(\delta_{i k} p^{f}\right)+F_{i}^{D}+\alpha \rho^{s} g_{i} .
\end{aligned}
$$

Here, $i, k=1,2,3$. The Cartesian coordinate system we consider is at an angle $\theta$ with respect to the horizontal (see Figure 3). In these equations $\alpha$ is the particle volume fraction, $u$ the fluid velocity vector, $v$ the solids velocity vector, $\vec{g}$ the gravity vector, $T^{s}$ the solids stress tensor, $p^{f}$ is the fluid pressure, $F^{D}$ the generalized drag force and $\delta$ represents the Kronecker delta function. 


\section{References}

[1] B. Akers and O. Bokhove, Hydraulic flow through a channel contraction: Multiple steady states, Phys. Fluids, 20 (2008) 056601.

[2] V.R. Ambati and O. Bokhove, Space-time discontinuous Galerkin finite element method for shallow water flows, J. Comput. Appl. Math. 204 (2007) 452.

[3] V.R. Ambati and O. Bokhove, Space-time discontinuous Galerkin discretization of rotating shallow water equations, J. Comput. Phys. 225 (2007) 1233.

[4] N.J. Balmforth and R.R. Kerswell, Granular collapse in two dimensions, J. Fluid Mech. 538 (2005) 399.

[5] M. Castro, J.M. Gallardo and C. Parés, High order finite volume schemes based on reconstruction of states for solving hyperbolic systems with nonconservative products. Application to shallow-water systems, Math. Comput. 75 (2006) 1103.

[6] M.-C. Chiou, Y. Wang and K. Hutter, Influence of obstacles on rapid granular flows, Acta Mechanica 175 (2005) 105.

[7] B. Cockburn and C.W. Shu, The Runge-Kutta discontinuous Galerkin method for conservation laws V, J. Comput. Phys. 141 (1998) 199.

[8] G. Dal Maso, P.G. LeFloch and F. Murat, Definition and weak stability of nonconservative products, J. Math. Pures Appl. 74 (1995) 483.

[9] R.P. Denlinger and R.M. Iverson, Flow of variably fluidized granular masses across three-dimensional terrain 2. Numerical predictions and experimental tests, J. Geophys. Res. 106 (2001) 553.

[10] D.A. Drew and R.T. Lahey, Analytical modeling of multiphase flow, in Particulate Two-Phase flows, edited by M.C. Roco, Butterworth-Heinemann, Boston, 1993.

[11] J.M.N.T. Gray, Y.-C. Tai and S. Noelle, Shock waves, dead zones and particlefree regions in rapid granular free-surface flows, J. Fluid Mech. 491 (2003) 161.

[12] D.D. Houghton and A. Kasahara, Nonlinear Shallow Fluid Flow Over an Isolated Ridge, Comm. on Pure and Applied Math. 21 (1968) 1.

[13] K. Hutter and K.R. Rajagopal, On flows of granular materials, Continuum Mech. Thermodyn. 6 (1994) 81.

[14] R.M. Iverson and R.P. Denlinger, Flow of variably fluidized granular masses across three-dimensional terrain 1. Coulomb mixture theory, J. Geophys. Res. 106 (2001) 537.

[15] R. Jackson, The dynamics of fluidized particles, Cambridge University Press, 2000. 
[16] C.M. Klaij, J.J.W. van der Vegt and H. van der Ven, Space-time discontinuous Galerkin method for the compressible Navier-Stokes equations, J. Comput. Phys. 217 (2006) 589.

[17] L. Krivodonova, J. Xin, J.F. Remacle, N. Chevaugeon and J.E. Flaherty, Shock detection and limiting with discontinuous Galerkin methods for hyperbolic conservation laws, Appl. Numer. Math. 48 (2004) 323.

[18] L.H. Le, New models for geophysical flows, Ph. D. dissertation, State University of New York at Buffalo, 2006.

[19] R.J. LeVeque, Balancing source terms and flux gradients in high-resolution Godunov methods: the quasi-steady wave-propagation algorithm, J. Comput. Phys., 146 (1998) 346.

[20] J. Ling, P.V. Skudarnov, C.X. Lin and M.A. Ebadian, Numerical investigations of liquid-solid slurry flows in a fully developed turbulent flow region, International Journal of Heat and Fluid Flow 24 (2003) 389.

[21] H. Luo, J.D. Baum and R. Löhner, A Hermite WENO-based limiter for discontinuous Galerkin method on unstructured grids, J. Comput. Phys. 225 (2007) 686.

[22] J.J. Major and R.M. Iverson, Debris-flow deposition: Effects of pore-fluid pressure and friction concentrated at flow margins, GSA Bulletin 111 (1999) 1424.

[23] A.K. Patra, C.C. Nichita, A.C. Bauer, E.B. Pitman, M. Bursik and M.F. Sheridan, Parallel adaptive discontinuous Galerkin approximation for thin layer avalanche modeling, Computers and Geosciences 32 (2006) 912.

[24] A.K. Patra, A.C. Bauer, C.C. Nichita, E.B. Pitman, M.F. Sheridan, M. Bursik, B. Rupp, A. Webber, A.J. Stinton, L.M. Namikawa and C.S. Renschler, Parallel adaptive numerical simulation of dry avalanches over natural terrain, J. Volc. Geothermal Research 139 (2005) 1.

[25] E.B. Pitman and L. Le, A two-fluid model for avalanche and debris flows, Phi. Trans. R. Soc. A 363 (2005) 1573.

[26] O. Pouliquen and Y. Forterre, Friction law for dense granular flows: application to the motion of a mass down a rough inclined plane, J. Fluid Mech. 453 (2002) 133.

[27] S. Rhebergen, O. Bokhove and J.J.W. van der Vegt, Discontinuous Galerkin finite element methods for hyperbolic nonconservative partial differential equations, J. Comput. Phys. 227 (2008) 1887.

[28] S.B. Savage and K. Hutter, The dynamics of avalanches of granular materials down a rough incline, J. Fluid Mech. 199 (1989) 177.

[29] Y.C. Tai, S. Noelle, J.M.N.T. Gray and K. Hutter, Shock-capturing and fronttracking methods for granular avalanches, J. Comput. Phys 175 (2002) 269. 
[30] P.A. Tassi, O. Bokhove and C.A. Vionnet, Space discontinuous Galerkin method for shallow water flows - kinetic and HLLC flux, and potential vorticity generation, Adv. Water Resour. 30 (2007) 998.

[31] E.F. Toro, Riemann solvers and numerical methods for fluid dynamics, Springer Verlag, 1997.

[32] J.J.W. van der Vegt and H. van der Ven, Space-Time Discontinuous Galerkin Finite Element Method with Dynamic Grid Motion for Inviscid Compressible Flows I. General Formulation, J. Comput. Phys. 182 (2002) 546.

[33] A.W. Vreman, M. Al-Tarazi, J.A.M. Kuipers, M. van Sint Annaland and O. Bokhove, Supercritical shallow granular flow through a contraction, experiment, theory and simulation, J. Fluid Mech. 578 (2007) 233.

[34] Y. Wang, K. Hutter and S.P. Pudasaini, The Savage-Hutter theory: A system of partial differential equations for avalanche flows of snow, debris and mud, Z. Angew. Math. Mech. 84 (2004) 507.

[35] Y. Xing and C.W. Shu, High order well-balanced finite volume WENO schemes and discontinuous Galerkin methods for a class of hyperbolic systems with source terms, J. Comput. Phys. 214 (2006) 567.

[36] http://www.liceng.dk/LIC/Services/SlurryAndSediment/index.shtml

[37] http://volcanoes.usgs.gov/images/pglossary/lahar.php 